\title{
Portfólio E-V eficiente: Bibliometria das evoluções considerando simulação ou métricas do risco com espaço objetivo aumentado
}

\author{
Portfolio E-V efficient: Bibliometrics of developments considering simulation or risk metrics with \\ increased objective space \\ Portafolio E-V eficiente: Bibliometría de desarrollos considerando simulación o métricas de riesgo \\ con espacio objetivo aumentado
}

\author{
Marcos Huber Mendes \\ ORCID: https://orcid.org/0000-0002-7203-3552 \\ Pontifícia Universidade Católica do Rio de Janeiro, Brasil \\ E-mail: hubermendes@decisionsupport.com.br \\ Reinaldo Castro Souza \\ ORCID: https://orcid.org/0000-0002-0210-3703 \\ Pontifícia Universidade Católica do Rio de Janeiro, Brasil \\ E-mail: reinaldo@puc-rio.br \\ Marco Aurélio Sanfins \\ ORCID: https://orcid.org/0000-0001-9807-1541 \\ Universidade Federal Fluminense, Brasil \\ E-mail: marcosanfins@id.uff.br \\ Tuany Esthefany Barcellos de Carvalho Silva \\ ORCID: https://orcid.org/0000-0003-4543-9116 \\ Pontifícia Universidade Católica do Rio de Janeiro, Brasil \\ E-mail: tuanybarcellos@aluno.puc-rio.br \\ Lucas Mattos Martins \\ ORCID: https://orcid.org/0000-0002-2677-3277 \\ Universidade Federal Fluminense, Brasil \\ E-mail: lucasmattosmartins@id.uff.br
}

\begin{abstract}
Resumo
A produção científica e a necessidade de indicadores que a quantifique se fortaleceu e cresceu nas últimas décadas, como também a de ferramentas que auxiliem na sua mensuração e a busca por técnicas para analisar de forma temporal, entre outras, o volume de publicações, autores, citações e citações das referências. A bibliometria é uma importante técnica quantitativa e estatística para medir índices de produção e conhecimento científico utilizando softwares, palavras chaves de interesse e títulos para realizar levantamentos bibliométricos em plataformas de repositórios de artigos científicos, teses e livros. O trabalho objetiva analisar a veracidade de duas suposições sobre as evoluções, até a presente data, do modelo da teoria de portfólio E-V eficiente de Markowitz. A primeira se existe evoluções do modelo, com simulação pelo método de Monte Carlo, que utilizem a simulação como um fim para obter a distribuição de probabilidade empírica de todos os resultados ótimos inerentes à simulação e não, pelo usual método de concentração de dados, sendo apenas um meio para se obter melhores resultados e modelos, para comparação entre modelos ou para análise de resultados dos modelos ou ainda para projeção dos resultados dos modelos em janelas de holdout. A segunda se existe evoluções do modelo utilizando métricas do risco com espaço objetivo aumentado, possibilitando a estimação de um maior número de parâmetros. A partir de softwares para análise bibliométrica se conseguiu chegar à conclusão de que na teoria analisada, e nas suas evoluções até a presente data, as duas suposições não são verdadeiras.

Palavras chave: Portfólio E-V eficiente; Otimização; Simulação; Monte carlo; Distribuição de probabilidade empírica; Métrica do risco; Espaço objetivo aumentado; Bibliometria.
\end{abstract}

\begin{abstract}
Scientific production and the need for indicators that quantify it have strengthened and grown in the last decades, as well as tools that help in their measurement and the search for techniques to analyze in a temporal way, among others, the volume of publications, authors, citations and citations of references. Bibliometry is an important quantitative and statistical technique for measuring production indexes and scientific knowledge using software, keywords of interest and titles to carry out bibliometrics surveys on platforms for repositories of scientific articles, theses and books. The work aims to analyze the veracity of two assumptions about the evolution, to date, of Markowitz's theory of portfolio model E-V efficient. The first is whether there are evolutions of the model, with simulation by the Monte Carlo method, that use simulation as an end to obtain the empirical probability distribution of all the optimal results inherent to the
\end{abstract}


simulation and not, by the usual method of data concentration, being just a means to obtain better results and models, to compare models or to analyze the results of the models or even to project the results of the models in holdout windows. The second is whether there are evolutions of the model using risk metrics with increased objective space, allowing the estimation of a greater number of parameters. From software for bibliometric analysis it was possible to reach the conclusion that in the analyzed theory, and in its evolution to date, the two assumptions are not true.

Keywords: Portfolio E-V efficient; Optimization; Simulation; Monte Carlo; Empirical probability distribution; Risk metric; Increased objective space; Bibliometry.

\section{Resumen}

La producción científica y la necesidad de indicadores que la cuantifiquen se han fortalecido y crecido en las últimas décadas, así como herramientas que ayuden en su medición y la búsqueda de técnicas para analizar de manera temporal, entre otros, el volumen de publicaciones, autores, citas y citas de referencias. La bibliometría es una técnica cuantitativa y estadística importante para medir índices de producción y conocimiento científico utilizando software, palabras clave de interés y títulos para realizar encuestas bibliométricas en plataformas para repositorios de artículos científicos, tesis y libros. El trabajo tiene como objetivo analizar la veracidad de dos supuestos sobre la evolución, hasta la fecha, de lo modelo de Markowitz de la teoría de portfolio E-V eficiente. La primera es que existen evoluciones del modelo, con simulación por el método de Monte Carlo, que utilizan la simulación como fin para obtener la distribución de probabilidad empírica de todos los resultados óptimos inherentes a la simulación y no, por el método habitual de concentración de datos, siendo solo un medio para obtener mejores resultados y modelos, comparar modelos o analizar los resultados de los modelos o incluso proyectar los resultados de los modelos en ventanas de holdout. La segunda es que existen evoluciones del modelo utilizando métricas de riesgo con espacio objetivo aumentado, permitiendo la estimación de un mayor número de parámetros. A partir de un software de análisis bibliométrico, pudimos llegar a la conclusión de que en la teoría analizada, y en su evolución hasta la fecha, los dos supuestos no son ciertos.

Palabras clave: Portfolio E-V eficiente; Optimización; Simulación; Monte carlo; Distribución de probabilidad empírica; Métrica de riesgo; Espacio objetivo aumentado; Bibliometría.

\section{Introdução}

A busca por conhecimento é inerente ao ser humano. Desde os primórdios da civilização, os indivíduos buscam pelo saber, como produzir e guardar novas informações. Atualmente é essencial a busca por ferramentas que auxiliam pesquisadores a obter as informações desejadas sobre temas específicos. Com o avanço tecnológico surgiram ferramentas inovadoras que possibilitam a busca ampla e temporal de assuntos pontuais, uma metodologia muito utilizada no Brasil, a bibliometria. (Chueke, 2015).

A bibliometria pode ser definida como um conjunto de regras que, quando aplicadas junto a métodos quantitativos, buscam organizar, estruturar e disponibilizar a produção científica encontrada em periódicos, autores e em outras apresentações da informação. Essas técnicas quantitativas e estatísticas também podem ser utilizadas a fim de medir os índices de divulgação de materiais científicos (Andre 2012). O estudo bibliométrico tem como finalidade principal a criação de indicadores que possibilitam sumarizar as publicações científicas de instituições, de autores mais buscados, das áreas de estudo mais requeridas e dos países que mais produzem conteúdo científico, entre outros, conforme (Silva, 2016). Pesquisas apontam que o aumento da procura por bibliometria se deve ao fato de que nas últimas décadas os avanços tecnológicos e o acesso à internet trouxeram facilidades na propagação das informações. Assim, desenvolveu-se programas e algoritmos a fim de criar bases de dados, possibilitando o cruzamento das informações de diversas variáveis, a fim de levantar estatísticas a respeito dos conhecimentos científicos (Pimenta, 2017).

Cada vez mais se procura atualizar ou implantar softwares que sejam úteis para os produtores de conhecimento. As ferramentas, que se desenvolvem constantemente, procuram atender as mais variadas necessidades. Atualmente se pode citar como exemplo de bases para busca dentro da web a Scopus, a Web of Science, a Tycho e o Instituto Nacional de Propriedade Industrial (INPI). Segundo Chueke (2015), seria simplório afirmar que dentro de um conhecimento específico não há a presença de novos desafios a serem resolvidos e que tais desafios estariam prontos para serem colocados em prática e serem difundidos. É grande a importância da realização de pesquisas no meio acadêmico, a fïm de possibilitar a evolução dos mais variados estudos, sendo eles novos ou antigos, porém, ainda sem solução. Dessa forma, propiciando o avanço da produção de novos saberes. 
Os estudos bibliométricos corroboram para as pesquisas científicas. Alinhado o foco da pesquisa, este método ajuda a dimensionar e direcionar a área de conhecimento, de estudos e de problemas no qual o pesquisador está buscando aprimoramento. Dentro deste processo é possível analisar e ter acesso as literaturas mais relevantes, mapear os conceitos mais profundos, os autores que mais obtiveram sucesso nesta área da ciência e ainda conseguir levantar dados sobre métodos adequados já utilizados em outros trabalhos (Chueke, 2015). Este trabalho realiza uma pesquisa bibliométrica que objetiva analisar duas suposições: primeiro se na teoria de portfólio E-V eficiente de Markowitz (1952) e nas suas evoluções até a presente data, existem modelos de simulação pelo método de Monte Carlo, que utilizem a simulação como um fim para obter a distribuição de frequência de todos os resultados ótimos inerentes à simulação e segundo se na teoria de portfólio E-V eficiente de Markowitz (1952) e nas suas evoluções até a presente data, existem modelos utilizando métricas do risco com espaço objetivo aumentado, possibilitando a estimação de um maior número de parâmetros e, assim, a busca por melhores resultados. Foi utilizada a base de dados da Scopus e da Web of Science por meio de palavras chaves, de acordo com o objetivo que se pretende alcançar. O banco de dados da Scopus é o maior banco de dados de resumos e citações da literatura revisada por pares, com ferramentas bibliométricas para monitorar, analisar e visualizar a pesquisa.

\section{Metodologia}

A pesquisa bibliométrica e o conjunto de palavras chaves de busca foram orientadas conforme o artigo A comprehensive review of deterministic models and applications for mean-variance portfolio optimization, Kalayci, C. B. (2019). De acordo com (Wallin, 2012) a análise bibliométrica da produção científica de uma área do conhecimento aumenta a objetividade do pesquisador ao permitir a análise de grandes quantidades de dados e ao identificar relacionamentos difíceis de serem detectados sem o auxílio de computadores, e de métodos estatísticos.

O presente trabalho segundo Gil, A. C, (2008) pode ser considerado como:

- Descritivo - ao pesquisar e descrever a evolução de um determinado método;

- Dedutivo - pois parte de princípios reconhecidos como verdadeiros e indiscutíveis, chegando a conclusões de maneira lógica, considerando que as premissas obtidas para o seu desenvolvimento são generalizadas;

- Aplicado - pois objetiva aplicação prática dos conhecimentos gerais a situações específicas;

- Pesquisa quantitativa - pois requer o uso de recursos computacionais e de técnicas estatísticas para seleção automática de dados e informações;

- Pesquisa qualitativa - pois também recorre a seleção e classificação sem a utilização de técnicas quantitativas;

- Pesquisa exploratória - de acordo com seus objetivos, tendo como preocupação central identificar os fatores que determinam ou que contribuem para a ocorrência dos fenômenos analisados;

e utiliza a coleta de dados secundários a partir dos repositórios de dados da Scopus e da Web of Science.

Segundo Domingues (2018), "a criação da bibliometria e seu estabelecimento como ferramenta matemática para dimensionamento da produção científica foram estabelecidas por Otlet (1934) na sua obra "Traité de documentatión: Le Livre Sur Le Livre" e difundidas por Pritchard (1969) em sua publicação que questiona o uso do termo "Bibliografia Estatística" ou "Bibliometria". Vanti (2002) mostra as características e interações entre a bibliometria, a cienciometria e a informetria incluindo a webometria como mecanismos utilizados para medir o registro da informação e a difusão do conhecimento, Araújo, C. A, (2006) estuda a evolução histórica da bibliometria".

É relevante ressaltar o valor inestimável de quantificar o "saber", os estudos bibliométricos apresentam métricas robustas com a finalidade de esclarecer de forma ampla e completa o quão importante é sumarizar as buscas por temas pontuais. A criação de indicadores é primordial para alcançar os objetivos por meio de uma busca bibliométrica, é a partir destes indicadores que se consegue ter as informações necessárias para a conclusão do estudo (Penteado, 2002). 
Penteado (2002), afirma que para fazer uma manipulação automatizada dos dados se deve seguir quatro etapas, sendo elas a identificação das necessidades de informação, a preparação dos dados, o tratamento bibliométrico e a análise dos resultados. É desta forma que se pode realizar a investigação dos dados com o auxílio de um software que faça análises bibliométricas. A bibliometria pode apresentar o registro de toda a produção científica de um determinado assunto. $\mathrm{O}$ uso de tecnologia é indispensável para a criação de um repositório de material científico, assim como para o mapeamento da produção científica. Dessa forma, existem diferentes métodos de pesquisa que se adequam conforme o propósito do estudo bibliométrico. A investigação pode ser feita a partir de palavras chaves ou da quantidade de determinada palavra no título ou no resumo das publicações, entre outras informações (Soares, 2016).

A análise bibliométrica foi elaborada com base nos dados pesquisados na Scopus e na Web of Science utilizando o software R com o pacote Bibliometrix, Aria e Cuccurullo, (2017), para gerar os Gráfico, Figuras e Tabelas a respeito das pesquisas realizadas.

\section{Resultados e Discussão}

Foram realizadas seis pesquisas bibliométricas utilizando três conjuntos de palavras chaves, sendo três pesquisas na base de dados da Scopus e três pesquisas na base de dados da Web of Science.

O banco de dados da Scopus é o maior banco de dados de resumos e citações da literatura revisada por pares, com ferramentas bibliométricas para monitorar, analisar e visualizar a pesquisa. Segundo informação solicitada a Scopus esta dispõe atualmente de:

- $\quad 80$ milhões de resultados vindos de 24.272 títulos seriais cobertos, 101.000 conferências e 230.000 livros;

- $\quad$ Conteúdo publicado por mais de 5.000 editores em 105 países e mais de 40 idiomas;

- $\quad$ Atualizada diariamente - aproximadamente 10.000 novos documentos indexados por dia;

- $\quad$ 10.807.000 documentos de acesso aberto;

- $\quad$ Cobertura de "articles in press" de mais de 8.075 títulos.

Segundo o site da Web of Science esta dispõe de "estatísticas de tendências científicas extraídas de mais de 12 milhões de artigos de mais de 12.000 periódicos globais, o Essential Science Indicators oferece a cobertura detalhada que seja necessária para efetivamente analisar e comparar o desempenho da pesquisa, identificar tendências significativas, classificar os melhores desempenhos e avaliar funcionários e colaboradores em potencial. Com base em um arquivo contínuo de 10 anos, os indicadores Essential Science são atualizados a cada dois meses".

Para obter os objetivos desejados, serão realizadas seis pesquisa bibliométricas caracterizadas pelos repositórios utilizados e por um conjunto de palavras chaves. As pesquisas com o primeiro conjunto de palavras chaves é uma pesquisa generalizada sobre as extensões da teoria de portfólios E-V eficientes de Markowitz (1952), inclusive para modelos de simulação pelo método de Monte Carlo e para modelos com diferentes métricas do risco, e foi realizada com as seguintes palavras chave: TITLE-ABS-Key (Portfolio AND (selection OR management OR optimization) AND (mean OR variance)), para publicações no período a partir do ano de 2009, nos repositórios da Scopus e da Web of Science. As pesquisas com o segundo conjunto de palavras chaves é uma pesquisa especifica sobre as extensões da teoria de portfólios E-V eficientes de Markowitz (1952) para modelos de simulação pelo método de Monte Carlo, e foi realizada com as seguintes palavras chave: TITLE-ABS-Key (Portfolio AND ((selection OR management OR optimization) AND (mean OR variance)) AND (sampled OR sampling OR resampled OR \{monte carlo\} OR sample OR simulation)), para publicações no período a partir do ano de 2009, nos repositórios da Scopus e da Web of Science. E as pesquisas com o terceiro conjunto de palavras chaves é especifica sobre as extensões da teoria de portfólios E-V eficientes de Markowitz (1952) para modelos com diferentes métricas do risco, e foi realizada com as seguintes palavras chave: TITLE-ABS-Key (Portfolio AND ((selection OR management OR optimization) AND (mean OR variance)) AND 
(assessment OR metrics OR measures OR estimation)), também para publicações no período a partir do ano de 2009, nos repositórios da Scopus e da Web of Science.

As pesquisas realizadas na Scopus retornaram para o primeiro, o segundo e o terceiro conjunto de palavras chave, respectivamente, 1583 documentos, 376 documentos e 572 documentos, no período analisado. As pesquisas realizadas na Web of Science retornaram para o primeiro, o segundo e o terceiro conjunto de palavras chave, respectivamente, 355 documentos, 80 documentos e 567 documentos, no período analisado.

A análise bibliométrica será realizada em três etapas:

- $\quad$ Na primeira Etapa serão apresentados os sumários das três pesquisas bibliográficas realizadas na Scopus, por serem as pesquisas que possuem maior número de documentos;

- $\quad$ Na segunda etapa serão detalhados os resultados das três pesquisas realizadas na Scopus, também por serem as pesquisas que possuem maior número de documentos;

- $\quad$ Na terceira etapa serão examinados e classificados em conjunto além de todos os resultados de documentos das três pesquisas bibliométricas realizadas na base de dados da Scopus também todos os resultados de documentos das três pesquisas bibliométricas realizadas na base de dados da Web of Science. Serão examinados e classificados os campos de keywords, keywords plus (keywords das referências), abstract e title. Primeiro especificamente para as keywords sampled, sampling, resampled, monte carlo, sample e simulation, de forma que se possa concluir se existe algum documento a respeito de otimização para seleção de portfólios que apresente como resultado a distribuição da probabilidade empírica de todos os resultados ótimos de um modelo de otimização com simulação, e não pelo usual método de concentração de dados (por exemplo média e variância), e caso existam de que forma estes resultados seriam tratados nos documentos em questão. E segundo especificamente para as keyword assessment, metrics, measures e estimation, de forma que se possa concluir se existe algum documento a respeito de métrica do risco que apresente como resultado uma métrica do risco com espaço objetivo aumentado, de forma a considerar um maior número de parâmetros a serem otimizados, possibilitando, assim, a busca por melhores resultados. 
Primeira Etapa - Sumários das pesquisas realizadas na Scopus.

O sumário bibliométrico das pesquisas com o primeiro, o segundo e o terceiro conjuntos de palavras chaves, realizadas na Scopus, são apresentados a seguir, respectivamente nas Tabela 1, Tabela 2 e Tabela 3.

Tabela 1. Sumário da pesquisa na Scopus com o primeiro conjunto de palavras chaves.

\begin{tabular}{|c|c|}
\hline Description & Results \\
\hline \multicolumn{2}{|l|}{ MAIN INFORMATION ABOUT DATA } \\
\hline Timespan & 2010:2021 \\
\hline Sources (Journals, Books, etc) & 674 \\
\hline Documents & 1583 \\
\hline Average years from publication & 4,67 \\
\hline Average citations per documents & 7,962 \\
\hline Average citations per year per doc & 1,289 \\
\hline References & 43065 \\
\hline \multicolumn{2}{|l|}{ DOCUMENT TYPES } \\
\hline article & 1251 \\
\hline article in press & 2 \\
\hline book & 6 \\
\hline book chapter & 56 \\
\hline conference paper & 234 \\
\hline conference review & 9 \\
\hline data paper & 1 \\
\hline editorial & 2 \\
\hline erratum & 1 \\
\hline letter & 1 \\
\hline review & 19 \\
\hline short survey & 1 \\
\hline \multicolumn{2}{|l|}{ DOCUMENT CONTENTS } \\
\hline Keywords Plus (ID) & 3527 \\
\hline Author's Keywords (DE) & 3622 \\
\hline \multicolumn{2}{|l|}{ AUTHORS } \\
\hline Authors & 2628 \\
\hline Author Appearances & 4100 \\
\hline Authors of single-authored documents & 193 \\
\hline Authors of multi-authored documents & 2435 \\
\hline \multicolumn{2}{|l|}{ AUTHORS COLLABORATION } \\
\hline Single-authored documents & 236 \\
\hline Documents per Author & 0,602 \\
\hline Authors per Document & 1,66 \\
\hline Co-Authors per Documents & 2,59 \\
\hline Collaboration Index & 1,81 \\
\hline
\end{tabular}

Fonte: Dados da Scopus elaborado pelo autor.

A Tabela 1 acima apresenta o sumário da pesquisa na Scopus com o primeiro conjunto de palavras chaves, podemos perceber, entre outros, um total de 1583 documentos, uma média de 7,962 citações por documentos e um total de 43.065 referências. 
Tabela 2. Sumário da pesquisa na Scopus com o segundo conjunto de palavras chaves.

\begin{tabular}{l|r}
\hline Description & Results \\
\hline MAIN INFORMATION ABOUT DATA & $2010: 2021$ \\
\hline Timespan & 236 \\
\hline Sources (Joumals, Books, etc) & 376 \\
\hline Documents & 4,35 \\
\hline Average years from publication & 7,657 \\
\hline Average citations per documents & 1,325 \\
\hline Average citations per year per doc & 11818 \\
\hline References & \\
\hline DOCUMENT TYPES & 310 \\
\hline article & 12 \\
\hline book chapter & 45 \\
\hline conference paper & 3 \\
\hline conference review & 6 \\
\hline review & 2,1 \\
\hline DOCUMENT CONTENTS & 2,59 \\
\hline Keywords Plus (ID) & 2,3 \\
\hline Author's Keywords (DE) & 1214 \\
\hline AUTHORS & 1043 \\
\hline Authors & 796 \\
\hline Author Appearances & 975 \\
\hline Authors of single-authored documents & \\
\hline Authors of multi-authored documents & \\
\hline AUTHORS COLLABORATION & \\
\hline Single-authored documents & \\
\hline Documents per Author & \\
\hline Authors per Document & \\
\hline Co-Authors per Documents & \\
\hline Collaboration Index & \\
\hline & \\
\hline
\end{tabular}

Fonte: Dados da Scopus elaborado pelo autor

A Tabela 2 acima apresenta o sumário da pesquisa na Scopus com o segundo conjunto de palavras chaves, podemos perceber, entre outros, um total de 376 documentos, uma média de 7,657 citações por documentos e um total de 11.818 referências. 
Research, Society and Development, v. 10, n. 4, e57310413802, 2021

(CC BY 4.0) | ISSN 2525-3409 | DOI: http://dx.doi.org/10.33448/rsd-v10i4.13802

Tabela 3. Sumário da pesquisa na Scopus com o terceiro conjunto de palavras chaves.

\begin{tabular}{|c|c|}
\hline Description & Results \\
\hline \multicolumn{2}{|l|}{ MAIN INFORMATION ABOUTDATA } \\
\hline Timespan & 2010:2021 \\
\hline Sources (Journals, Books, etc) & 322 \\
\hline Documents & 572 \\
\hline Average years from publication & 4,4 \\
\hline Average citations per documents & 8,691 \\
\hline \multicolumn{2}{|l|}{ Average citations per year per doc Inf } \\
\hline References & 18029 \\
\hline \multicolumn{2}{|l|}{ DOCUMENT TYPES } \\
\hline article & 468 \\
\hline article in press & 1 \\
\hline book & 2 \\
\hline book chapter & 24 \\
\hline conference paper & 67 \\
\hline conference review & 5 \\
\hline erratum & 1 \\
\hline review & 4 \\
\hline \multicolumn{2}{|l|}{ DOCUMENT CONTENTS } \\
\hline Keywords Plus (ID) & 1711 \\
\hline Author's Keywords (DE) & 1497 \\
\hline \multicolumn{2}{|l|}{ AUTHORS } \\
\hline Authors & 1208 \\
\hline Author Appearances & 1490 \\
\hline Authors of single-authored documents & 72 \\
\hline Authors of multi-authored documents & 1136 \\
\hline \multicolumn{2}{|l|}{ AUTHORS COLLABORATION } \\
\hline Single-authored documents & 86 \\
\hline Documents per Author & 0,474 \\
\hline Authors per Document & 2,11 \\
\hline Co-Authors per Documents & 2,6 \\
\hline Collaboration Index & 2,34 \\
\hline
\end{tabular}

Fonte: Dados da Scopus elaborado pelo autor

A Tabela 3 acima apresenta o sumário da pesquisa na Scopus com o terceiro conjunto de palavras chaves, podemos perceber, entre outros, um total de 572 documentos, uma média de 8,691 citações por documentos e um total de 18.029 referências. 


\section{Segunda Etapa - Detalhamento das pesquisas realizadas na Scopus.}

$\mathrm{Na}$ segunda etapa serão detalhados os resultados das pesquisas com os três conjuntos de palavras chaves realizadas na Scopus. Será apresentado a seguir os resultados da pesquisa com o primeiro conjunto de palavras chaves iniciando pelo volume de produção anual no período da pesquisa conforme apresentado no Gráfico 1.

Gráfico 1. Produção Científica Anual - Scopus, primeiro conjunto de palavras chave.

\section{Annual Scientific Production}

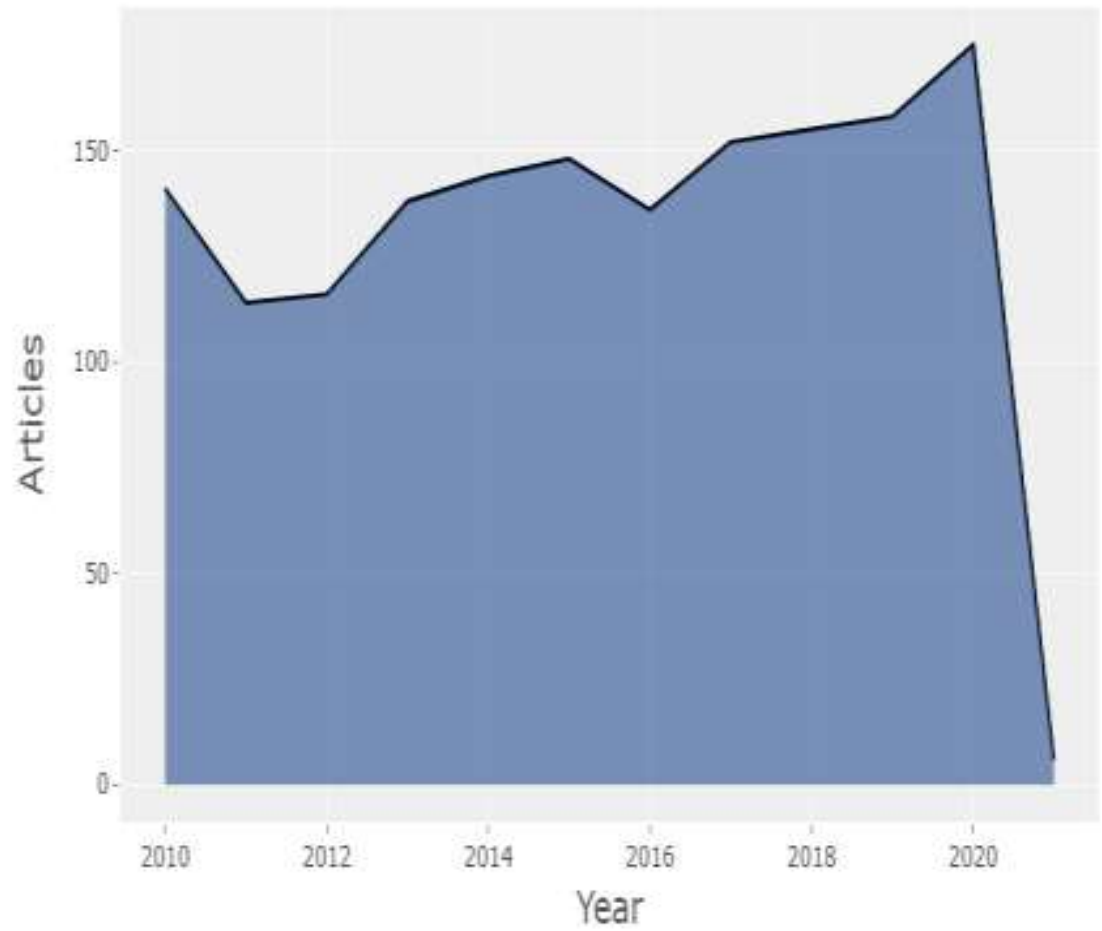

Fonte: Dados da Scopus elaborado pelo autor.

A pesquisa na Scopus com o primeiro conjunto de palavras chaves é uma pesquisa generalizada sobre as extensões da teoria de portfólios E-V eficientes de Markowitz (1952), inclusive modelos de simulação pelo método de Monte Carlo e métricas do risco, tem seu pico de produção em 2020 e um volume médio anual de aproximadamente cento e quarenta e cinco artigos científicos.

As vinte mais relevantes fontes utilizadas nas publicações da pesquisa na Scopus com o primeiro conjunto de palavras chaves são apresentadas no Gráfico 2 abaixo. 
Research, Society and Development, v. 10, n. 4, e57310413802, 2021

(CC BY 4.0) | ISSN 2525-3409 | DOI: http://dx.doi.org/10.33448/rsd-v10i4.13802

Gráfico 2. Fontes mais relevantes - Scopus, primeiro conjunto de palavras chave.

\section{Most Relevant Sources}

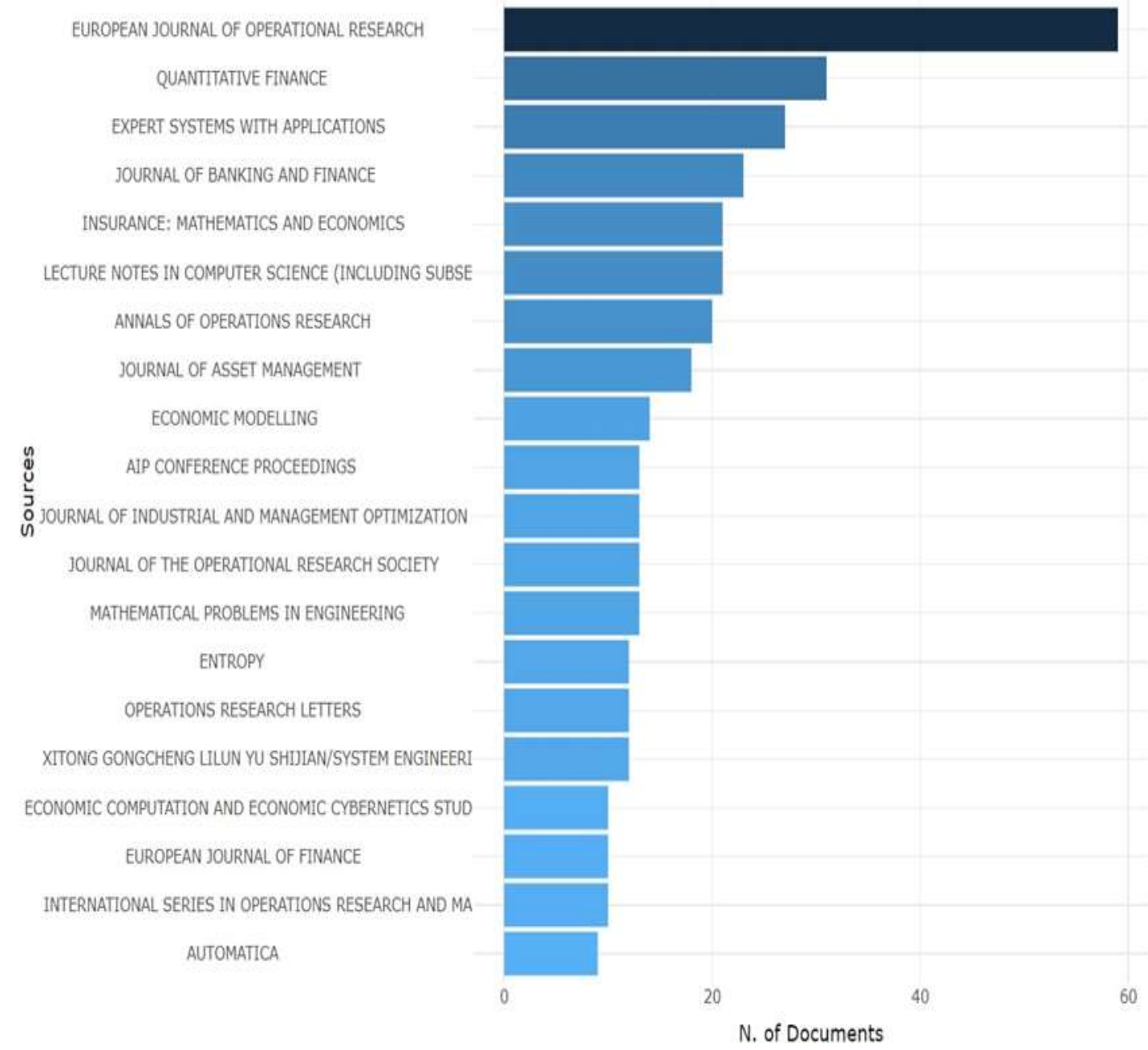

Fonte: Dados da Scopus elaborado pelo autor.

No Gráfico 2 acima podemos perceber que as fontes mais relevantes da pesquisa na Scopus com o primeiro conjunto de palavras chaves são o European Journal of Operational Research, o Quantitative Finance e o Expert Systems with Applications.

A Tabela 4 abaixo apresenta a classificação das trinta mais frequentes keywords utilizadas nas publicações da pesquisa na Scopus com o primeiro conjunto de palavras chaves. 
Tabela 4. Keywords mais frequentes - Scopus, primeiro conjunto de palavras chaves.

\begin{tabular}{|c|c|c|}
\hline & Words & Occurrences \\
\hline 1 & portfolio optimization & 217 \\
\hline 2 & portfolio selection & 199 \\
\hline 3 & efficient frontier & 72 \\
\hline 4 & mean-variance & 71 \\
\hline 5 & risk management & 48 \\
\hline 6 & mean-variance model & 44 \\
\hline 7 & mean-variance portfolio selection & 36 \\
\hline 8 & optimization & 36 \\
\hline 9 & portfolio & 36 \\
\hline 10 & dynamic programming & 34 \\
\hline 11 & asset allocation & 30 \\
\hline 12 & mean-variance optimization & 28 \\
\hline 13 & portfolio theory & 27 \\
\hline 14 & genetic algorithm & 26 \\
\hline 15 & portfolio optimisation & 26 \\
\hline 16 & sharpe ratio & 26 \\
\hline 17 & risk & 24 \\
\hline 18 & robust optimization & 24 \\
\hline 19 & transaction costs & 23 \\
\hline 20 & mean-variance analysis & 22 \\
\hline 21 & portfolio management & 22 \\
\hline 22 & optimal portfolio & 19 \\
\hline 23 & finance & 17 \\
\hline 24 & investment analysis & 17 \\
\hline 25 & multi-period portfolio selection & 16 \\
\hline 26 & portfolio choice & 16 \\
\hline 27 & regime switching & 16 \\
\hline 28 & stochastic control & 16 \\
\hline 29 & entropy & 15 \\
\hline 30 & hjb equation & 15 \\
\hline
\end{tabular}

Fonte: Dados da Scopus elaborado pelo autor.

$\mathrm{Na}$ Tabela 4 acima as keywords sampled, sampling, resampled, Monte Carlo, sample e simulation (segundo conjunto de palavras chaves) não aparecem na tabela, porém, aparecem na classificação total das mais frequentes keywords com a frequência total de 34 ocorrências. As keywords assessment, metrics, measures e estimation (terceiro conjunto de palavras chaves) não aparecem na tabela, porém, aparecem na classificação total das mais frequentes keywords com a frequência total de 37 ocorrências. Na classificação total das mais frequentes keywords não aparecem keywords relacionadas especificamente à modelos de otimização com simulação que apresentem a distribuição de frequência empírica dos ótimos simulados ou à métricas do risco com espaço objetivo aumentado, como evoluções do modelo de portfólio E-V eficiente. 
As Tabela 5 à Tabela 11 a seguir apresentam a classificação da frequência das duzentas e cinquenta keywords mais frequentes nas publicações da pesquisa na Scopus com o primeiro conjunto de palavras chaves classificadas por sete clusters: efficient frontier, optimization, mean-variance portfolio optimization, portfolio optimization, risk management, asset allocation e portfolio selection. As keywords relacionadas com a pesquisa na Scopus com o segundo conjunto de palavras chaves apresentam uma frequência total de 27 ocorrências. As keywords relacionadas com a pesquisa na Scopus com o terceiro conjunto de palavras chaves apresentam uma frequência total de 30 ocorrências. Nas duzentas e cinquenta keywords mais frequentes não aparecem keywords relacionadas especificamente à modelos de otimização com simulação que apresentem a distribuição de frequência empírica dos ótimos simulados ou à métricas do risco com espaço objetivo aumentado, como evoluções do modelo de portfólio E-V eficiente.

Tabela 5. Cluster Fronteira eficiente - Scopus, primeiro conjunto de palavras chaves.

\begin{tabular}{r|l|r|l}
\hline Cluster & efficient frontier & Cluster_label & l \\
\hline Occurrences & Words & Occurrences & Words \\
\hline 7 & cardinality & 6 & time-consistent strategy \\
\hline 71 & mean-variance & 26 & sharpe ratio \\
\hline 14 & asset-liability management & 8 & maximum principle \\
\hline 7 & mean-field formulation & 13 & mean-variance portfolio theory \\
\hline 7 & multi-period mean-variance model & 11 & hamilton-jacobi-bellman equation \\
\hline 7 & meanvariance portfolio selection & 15 & hib equation \\
\hline 16 & stochastic control & 16 & multi-period portfolio selection \\
\hline 10 & time inconsistency & 18 & optimal portfolio \\
\hline 72 & efficient frontier & 5 & multi-period mean-variance portfolio selection \\
\hline 8 & optimal portfolio selection & 6 & regime-switching \\
\hline 11 & mean-variance portfolio & 5 & constrained portfolio optimization \\
\hline 34 & dynamic programming & 6 & multiperiod portfolio optimization \\
\hline 16 & regime switching & 6 & viscosity solution \\
\hline 6 & time-inconsistency & 7 & uncertain exit time \\
\hline 8 & backward stochastic differential equation & 5 partial information \\
\hline 36 & mean-variance portfolio selection & 6 & shortage function \\
\hline 10 & optimal control & 5 & inflation \\
\hline 11 & stochastic maximum principle & 5 & mean-variance formulation \\
\hline 16 & portfolio choice & 8 & mean-variance criterion \\
\hline 9 & stochastic optimal control & & \\
\hline & &
\end{tabular}

Fonte: Dados da Scopus elaborado pelo autor.

$\mathrm{Na}$ Tabela 5 acima, cluster de Fronteira eficiente, não aparecem keywords relacionadas especificamente à modelos de otimização com simulação que apresentem a distribuição de frequência empírica dos ótimos simulados ou à métricas do risco com espaço objetivo aumentado, como evoluções do modelo de portfólio E-V eficiente. 
Research, Society and Development, v. 10, n. 4, e57310413802, 2021

(CC BY 4.0) | ISSN 2525-3409 | DOI: http://dx.doi.org/10.33448/rsd-v10i4.13802

Tabela 6. Cluster Otimização - Scopus, primeiro conjunto de palavras chaves.

\begin{tabular}{r|l|r|l}
\hline Cluster & optimization & Cluster_Label & 2 \\
\hline Occurrences & Words & Occurrences & Words \\
\hline 36 & optimization & 24 & risk \\
\hline 11 & uncertainty & 12 & investment \\
\hline 8 & simulation & 5 & clustering \\
\hline 10 & portfolio construction & 8 & portfolio performance \\
\hline 24 & robust optimization & 6 & covariance matrix \\
\hline 27 & portfolio theory & 6 & regularization \\
\hline 36 & portfolio & 10 & portfolio investment \\
\hline 6 & monte carlo simulation & 6 & mean variance optimization \\
\hline 12 & markowitz & 5 & linear programming \\
\hline 5 & lagrange multiplier & 5 & investments \\
\hline 9 & return & & \\
\hline
\end{tabular}

Fonte: Dados da Scopus elaborado pelo autor.

Na Tabela 6 acima, cluster de Otimização, a frequência em relação a frequência em relação as keywords simulation e Monte Carlo simulation é de respectivamente 8 e 6 ocorrências. Não aparecem keywords relacionadas especificamente à modelos de otimização com simulação que apresentem a distribuição de frequência empírica dos ótimos simulados ou à métricas do risco com espaço objetivo aumentado, como evoluções do modelo de portfólio E-V eficiente.

Tabela 7. Cluster Portfólio de otimização média-variância - Scopus, primeiro conjunto de palavras chaves.

\begin{tabular}{r|l|r|l}
\hline Cluster & mean-variance portfolio optimization & Cluster_Label & 3 \\
\hline Occurrences & Words & Occurrences & Words \\
\hline 10 & meanvariance model & 5 & factor model \\
\hline 5 & international diversification & 6 & renewable energy \\
\hline 12 & quadratic programming & 6 & branch-and-bound \\
\hline 15 & mean-variance portfolio optimization & 6 & systematic risk \\
\hline 9 & stochastic dominance & & \\
\hline
\end{tabular}

Fonte: Dados da Scopus elaborado pelo autor.

Na Tabela 7 acima, cluster de Portfólio de otimização média-variância, não aparecem keywords relacionadas especificamente à modelos de otimização com simulação que apresentem a distribuição de frequência empírica dos ótimos simulados ou à métricas do risco com espaço objetivo aumentado, como evoluções do modelo de portfólio E-V eficiente. 
Research, Society and Development, v. 10, n. 4, e57310413802, 2021

(CC BY 4.0) | ISSN 2525-3409 | DOI: http://dx.doi.org/10.33448/rsd-v10i4.13802

Tabela 8. Cluster Otimização de portfólio - Scopus, primeiro conjunto de palavras chaves.

\begin{tabular}{r|l|l|l}
\hline Cluster & portfolio optimization & Cluster_Label & 4 \\
\hline Occurrences & Words & Occurrences & Words \\
\hline 10 & machine learning & 9 & stochastic programming \\
\hline 217 & portfolio optimization & 11 & multiobjective optimization \\
\hline 9 & robustness & 5 & naive diversification \\
\hline 22 & mean-variance analysis & 6 & meanvariance optimization \\
\hline 5 & risk measures & 6 & g11 \\
\hline 7 & parameter uncertainty & 8 & cardinality constraints \\
\hline 6 & sparse portfolio & 6 & risk measure \\
\hline 7 & estimation error & 5 & gold \\
\hline 12 & downside risk & 7 & cardinality constraint \\
\hline 6 & semivariance & 5 & elliptical distributions \\
\hline 17 & investment analysis & 12 & conditional value-at-risk \\
\hline 23 & transaction costs & 6 & differential evolution \\
\hline 5 & expected shortfall & 5 & multi-objective \\
\hline 5 & local search & 6 & evolutionary algorithms \\
\hline
\end{tabular}

Fonte: Dados da Scopus elaborado pelo autor.

Na Tabela 8 acima, cluster de Otimização de portfólio, a frequência em relação as keywords risk measures e estimation error é de respectivamente 7 e 8 ocorrências. Não aparecem keywords relacionadas especificamente à modelos de otimização com simulação que apresentem a distribuição de frequência empírica dos ótimos simulados ou à métricas do risco com espaço objetivo aumentado, como evoluções do modelo de portfólio E-V eficiente.

Tabela 9. Cluster Gerenciamento do risco - Scopus, primeiro conjunto de palavras chaves.

\begin{tabular}{r|l|r|l}
\hline Cluster & risk management & Cluster_Label & 5 \\
\hline Occurrences & Words & Occurrences & Words \\
\hline 5 & capm & 8 & portfolio allocation \\
\hline 6 & decision making & 6 & portfolio analysis \\
\hline 22 & portfolio management & 5 & risk analysis \\
\hline 10 & estimation risk & 8 & active portfolio management \\
\hline 17 & finance & 8 & black-litterman model \\
\hline 5 & robust statistics & 5 & semi-variance \\
\hline 48 & risk management & 7 & electricity market \\
\hline 15 & value-at-risk & 5 & electricity markets \\
\hline 28 & mean-variance optimization & 5 & hedging \\
\hline 5 & non-normality & & \\
\hline
\end{tabular}

Fonte: Dados da Scopus elaborado pelo autor.

Na Tabela 9 acima, cluster de Gerenciamento do risco, a frequência em relação a keyword estimation risk é de 10 ocorrências. Não aparecem keywords relacionadas especificamente à modelos de otimização com simulação que apresentem a distribuição de frequência empírica dos ótimos simulados ou à métricas do risco com espaço objetivo aumentado, como evoluções do modelo de portfólio E-V eficiente. 
Tabela 10. Cluster Alocação de ativos - Scopus, primeiro conjunto de palavras chaves.

\begin{tabular}{|c|c|c|c|}
\hline Cluster & asset allocation & Cluster_Label & 6 \\
\hline Occurrences & Words & Occurrences & Words \\
\hline 6 & mean-variance optimisation & $\begin{array}{r}7 \\
\end{array}$ & kurtosis \\
\hline 26 & portfolio optimisation & 6 & mean \\
\hline 30 & asset allocation & 6 & conditional value at risk \\
\hline 7 & out-of-sample performance & 5 & asset management \\
\hline 8 & garch & 7 & copula \\
\hline 12 & portfolio diversification & 5 & utility function \\
\hline 11 & var & 9 & variance \\
\hline 14 & cvar & 5 & asset pricing \\
\hline 14 & skewness & 7 & markowitz model \\
\hline
\end{tabular}

Fonte: Dados da Scopus elaborado pelo autor.

Na Tabela 10 acima, cluster de Alocação de ativos, a frequência em relação a keyword out-of-sample performance é de 7 ocorrências. Não aparecem keywords relacionadas especificamente à modelos de otimização com simulação que apresentem a distribuição de frequência empírica dos ótimos simulados ou à métricas do risco com espaço objetivo aumentado, como evoluções do modelo de portfólio E-V eficiente.

Tabela 11. Cluster Seleção de portfólio - Scopus, primeiro conjunto de palavras chaves.

\begin{tabular}{r|l|l|l}
\hline Cluster & portfolio selection & Cluster_Label & 7 \\
\hline Occurrences & Words & Occurrences & Words \\
\hline 199 & portfolio selection & 8 & background risk \\
\hline 10 & meanvariance & 7 & uncertainty theory \\
\hline 11 & uncertain variable & 6 & mean-variance utility \\
\hline 6 & robust portfolio optimization & 8 & fuzzy portfolio selection \\
\hline 7 & genetic algorithms & 6 & possibility theory \\
\hline 14 & multi-objective optimization & 5 & robust estimation \\
\hline 5 & expected utility & 8 & robust portfolio \\
\hline 15 & particle swarm optimization & 8 & behavioral finance \\
\hline 44 & mean-variance model & 6 & portfolio selection problem \\
\hline 5 & investment risk & 11 & transaction cost \\
\hline 5 & value at risk & 5 & possibilistic mean \\
\hline 8 & short selling & 15 & entropy \\
\hline 26 & genetic algorithm & 6 & fuzzy number \\
\hline 14 & diversification & 6 & parametric quadratic programming \\
\hline 11 & modern portfolio theory & 6 & mean-variance-skewness model \\
\hline 9 & mean-variance efficiency & 6 & mean-variance approach \\
\hline 5 & socially responsible investment & 6 & markowitz mean-variance model \\
\hline 9 & efficient frontiers & 5 & credibility measure \\
\hline 9 & higher moments & & \\
\hline & &
\end{tabular}

Fonte: Dados da Scopus elaborado pelo autor.

Na Tabela 11 acima, cluster de Seleção de portfólio, a frequência em relação a keyword robust estimation é de 5 ocorrências. O modelo de robust estimation utiliza simulação, porém, apresenta os resultados pelo conceito de concentração de dados, ou seja, medidas de posição e de dispersão. Não aparecem keywords relacionadas especificamente à modelos de otimização com simulação que apresentem a distribuição de frequência empírica dos ótimos simulados ou à métricas do risco com espaço objetivo aumentado, como evoluções do modelo de portfólio E-V eficiente. 
Research, Society and Development, v. 10, n. 4, e57310413802, 2021

(CC BY 4.0) | ISSN 2525-3409 | DOI: http://dx.doi.org/10.33448/rsd-v10i4.13802

Na Figura 1 abaixo será apresentado o dendrograma das cinquenta mais frequentes keywords utilizadas na pesquisa Scopus com o primeiro conjunto de palavras chaves.

Figura 1. Dendrograma das keywords - Scopus, com o primeiro conjunto de palavras chave.

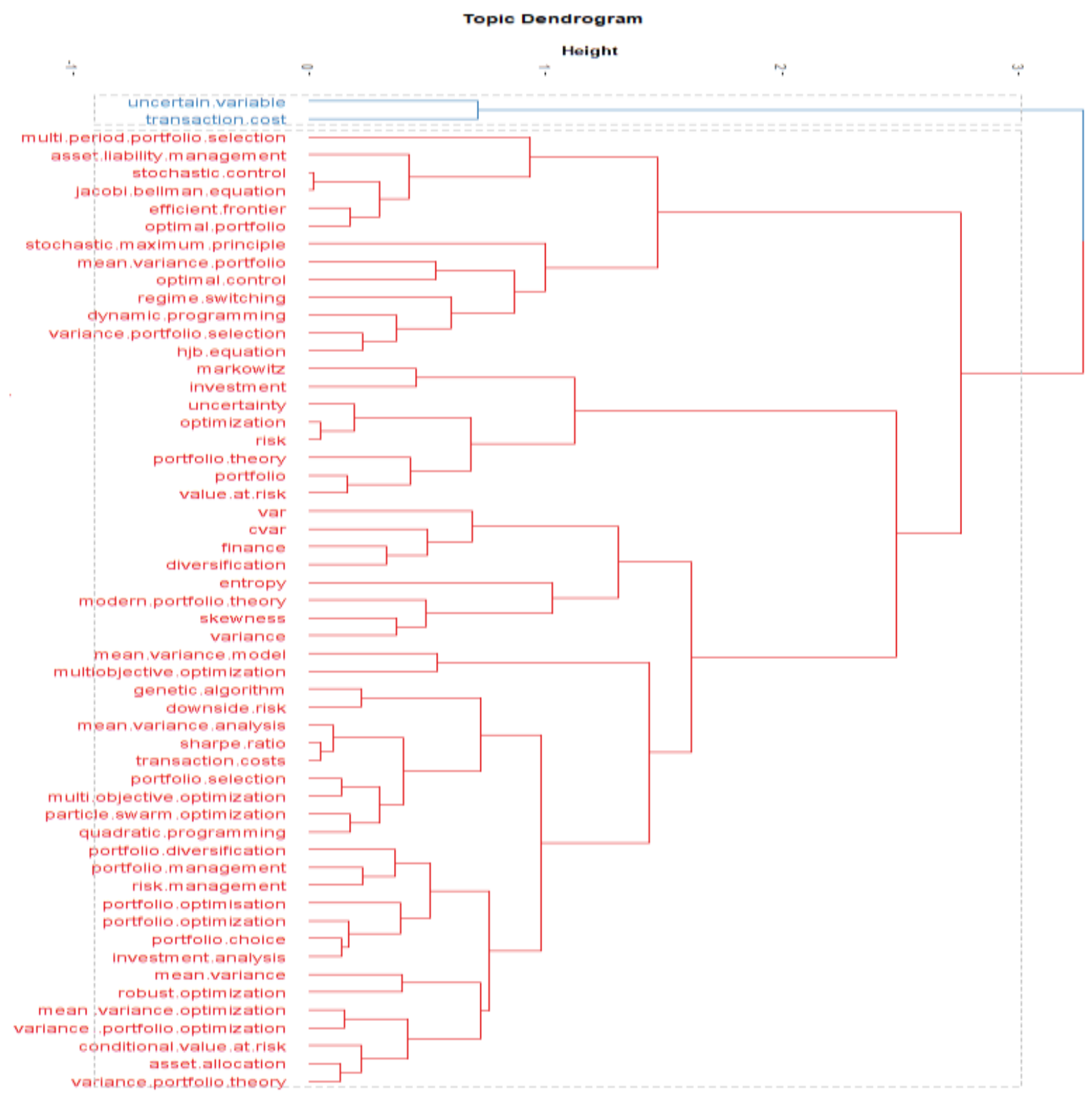

Fonte: Dados da Scopus elaborado pelo autor.

O dendrograma das cinquenta mais frequentes keywords, acima, apresenta dois grupos específicos, no segundo grupo estão incluídas as keywords value.at.risk, var, cvar, dowside.risk, sharp.ratio, robust.optimization, conditional value.at.risk, relativas ao segundo e ao terceiro conjunto de palavras chaves. Não aparecem keywords relacionadas especificamente à modelos de otimização com simulação que apresentem a distribuição de frequência empírica dos ótimos simulados ou à métricas do risco com espaço objetivo aumentado, como evoluções do modelo de portfólio E-V eficiente. 
Research, Society and Development, v. 10, n. 4, e57310413802, 2021

(CC BY 4.0) | ISSN 2525-3409 | DOI: http://dx.doi.org/10.33448/rsd-v10i4.13802

Na Figura 2 a seguir será apresentado o dendrograma das cinquenta mais frequentes keywords plus (mais frequentes keywords das referências) utilizadas na pesquisa da Scopus com o primeiro conjunto de palavras chaves.

Figura 2. Dendrograma das keywords plus - Scopus, primeiro conjunto de palavras chave.

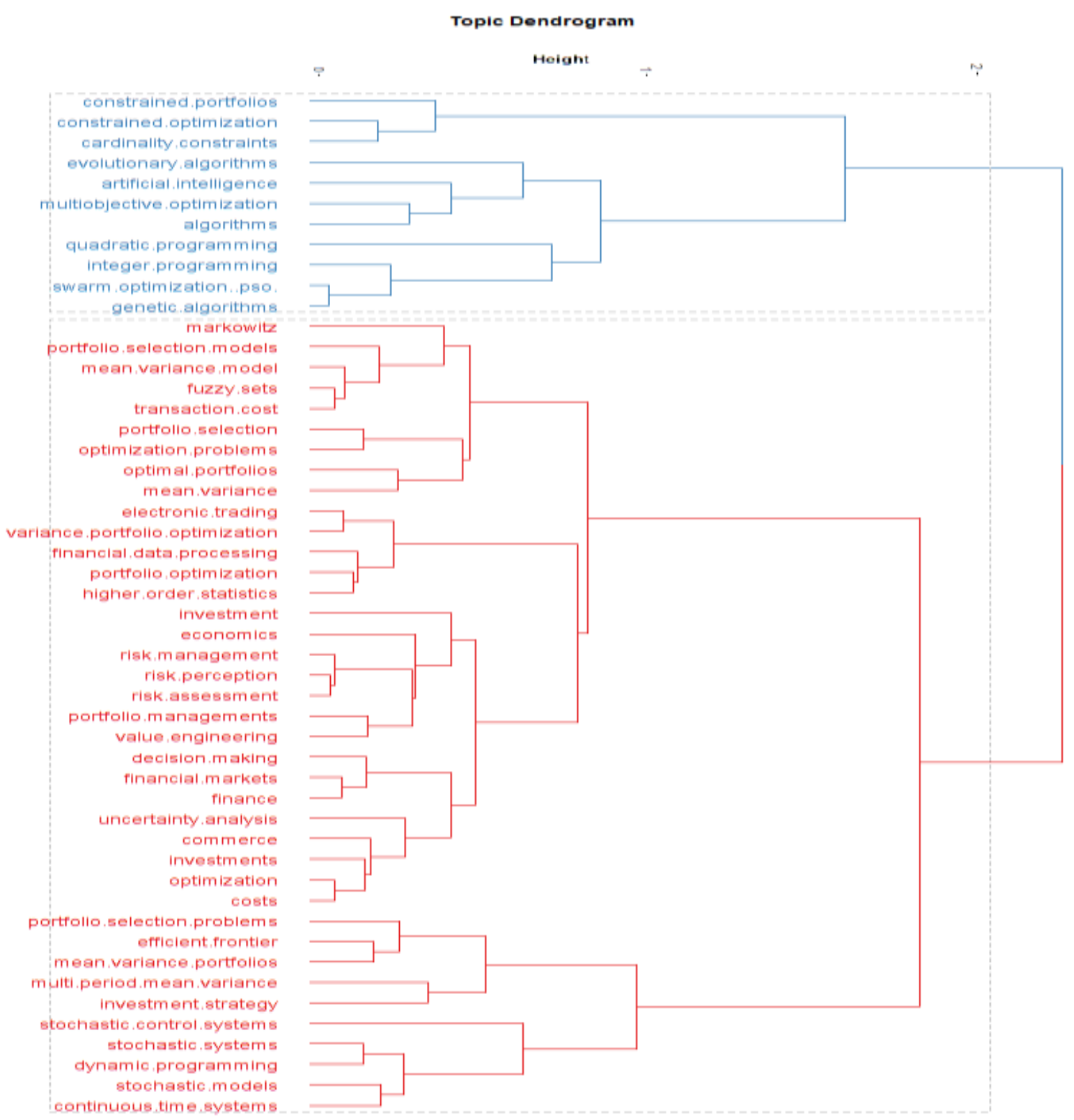

Fonte: Dados da Scopus elaborado pelo autor.

O dendrograma das cinquenta mais frequentes keywords plus, acima, apresenta dois grupos específicos. No segundo grupo está incluída a keyword higher.order.statistics, relativa ao terceiro conjunto de palavras chaves. Não aparecem keywords relacionadas especificamente à modelos de otimização com simulação que apresentem a distribuição de frequência empírica dos ótimos simulados ou à métricas do risco com espaço objetivo aumentado, como evoluções do modelo de portfólio E-V eficiente. 
Dando continuidade à Segunda Etapa da apresentação bibliométrica, serão apresentados a seguir os resultados da pesquisa na Scopus com o segundo conjunto de palavras chave iniciando pelo volume de produção anual no período conforme apresentado no Gráfico 3 abaixo.

Gráfico 3. Volume de produção anual - Scopus, segundo conjunto de palavras chave.

\section{Annual Scientific Production}

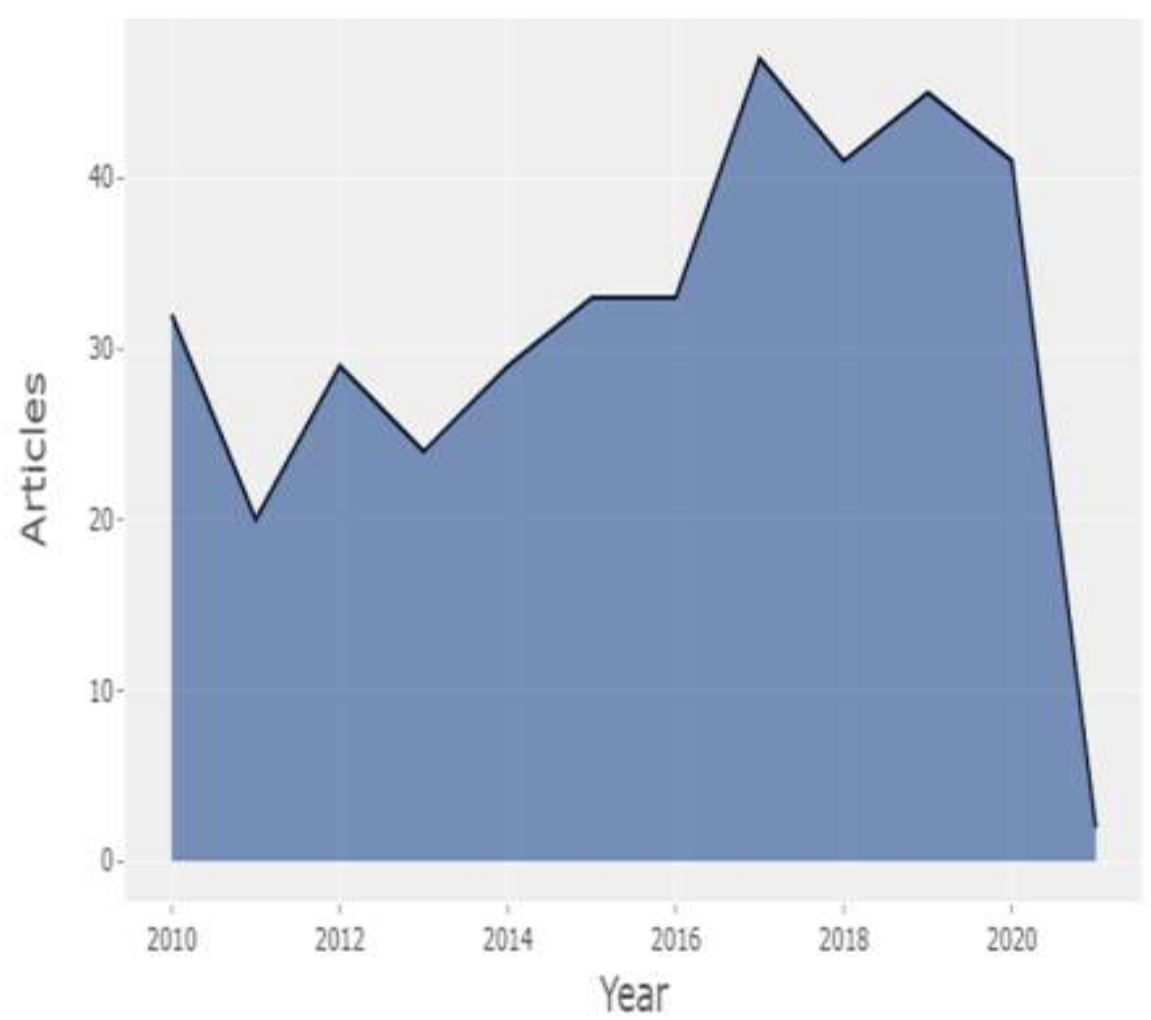

Fonte: Dados da Scopus elaborado pelo autor.

A pesquisa na Scopus com o primeiro conjunto de palavras chave é uma pesquisa generalizada sobre as extensões do modelo de portfólios E-V eficientes, inclusive métricas do risco e modelos de simulação pelo método de Monte Carlo e tem seu pico de produção em 2020 com um volume médio anual de aproximadamente cento e quarenta e cinco artigos científicos. Já a pesquisa na Scopus com o segundo conjunto de palavras chave é uma pesquisa específica sobre as extensões do modelo de portfólios E-V eficientes para modelos de simulação pelo método de Monte Carlo, tem seu pico de produção em 2017 e um volume médio anual de aproximadamente trinta e cinco artigos científicos.

As vinte mais relevantes fontes utilizadas nas publicações da pesquisa na Scopus com o segundo conjunto de palavras chave são apresentadas no Gráfico 4 abaixo. 
Research, Society and Development, v. 10, n. 4, e57310413802, 2021

(CC BY 4.0) | ISSN 2525-3409 | DOI: http://dx.doi.org/10.33448/rsd-v10i4.13802

Gráfico 4. Fontes mais relevantes - Scopus, segundo conjunto de palavras chave.

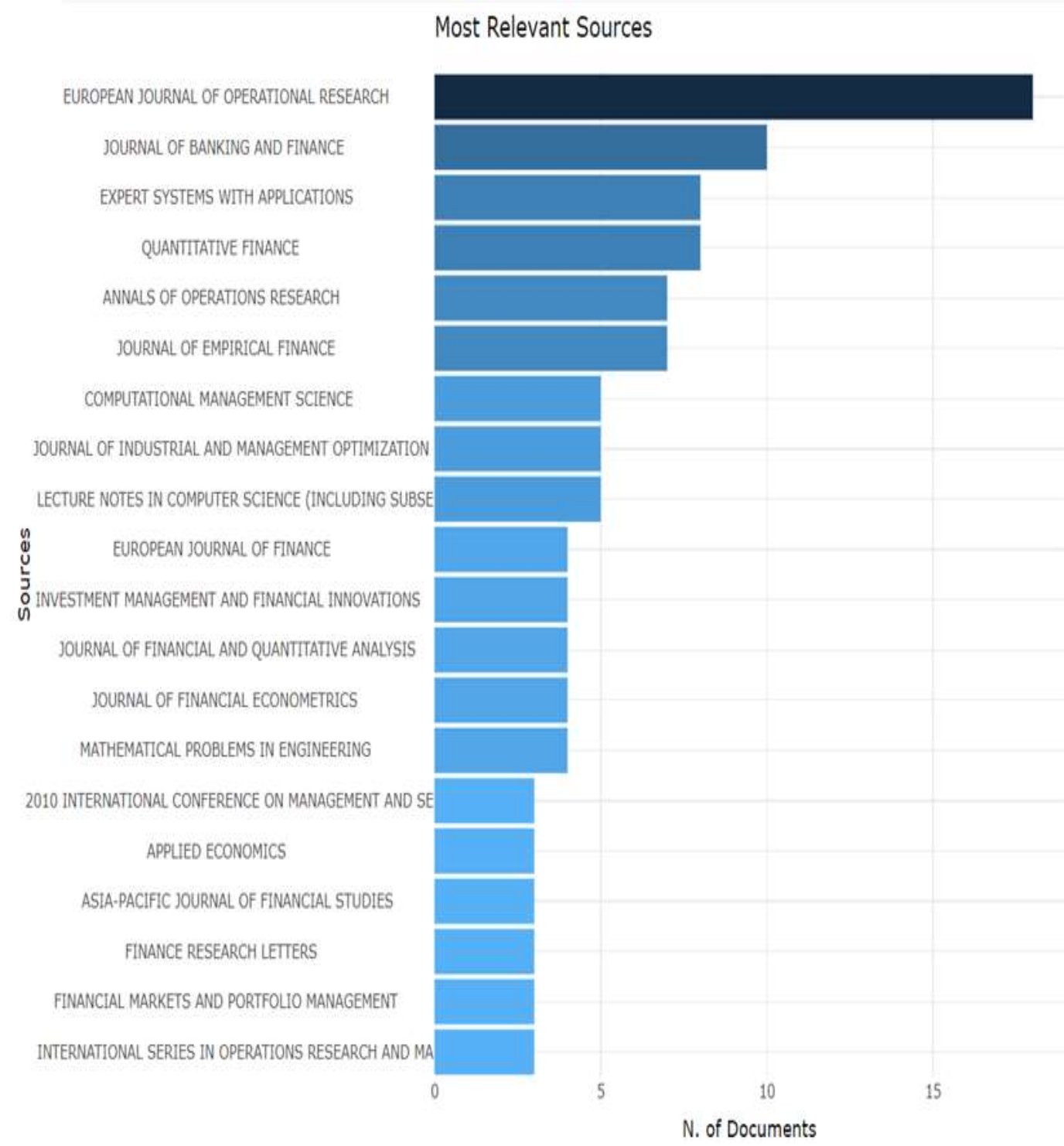

Fonte: Dados da Scopus elaborado pelo autor.

No Gráfico 4 acima podemos perceber que as fontes mais relevantes da pesquisa na Scopus com o segundo conjunto de palavras chaves são o European Journal of Operational Research, o Jounal of Bamk and Finance e o Expert Systems with Applications.

A Tabela 12 abaixo apresenta a classificação das trinta mais frequentes keywords utilizadas nas publicações da pesquisa na Scopus com o segundo conjunto de palavras chave. As keywords sampled, sampling, resampled, Monte Carlo, sample e simulation (segundo conjunto de palavras chaves) aparecem nas trinta mais frequentes keywords com 21 ocorrências e aparecem na classificação total das mais frequentes keywords com a frequência total de 65 ocorrências. As keywords assessment, metrics, measures e estimation (terceiro conjunto de palavras chaves) aparecem nas trinta mais frequentes keywords com 14 ocorrências e aparecem na classificação total das mais frequentes keywords com a frequência total de 30 ocorrências 
Tabela 12. Keywords mais frequentes - Scopus, segundo conjunto de palavras chave.

\begin{tabular}{|c|c|c|}
\hline & Words & Occurrences \\
\hline 1 & portfolio optimization & 62 \\
\hline 2 & portfolio selection & 40 \\
\hline 3 & risk management & 15 \\
\hline 4 & efficient frontier & 13 \\
\hline 5 & mean-variance & 13 \\
\hline 6 & asset allocation & 11 \\
\hline 7 & portfolio choice & 10 \\
\hline 8 & portfolio optimisation & 10 \\
\hline 9 & portfolio theory & 9 \\
\hline 10 & mean-variance optimization & 8 \\
\hline 11 & simulation & 8 \\
\hline 12 & copula & 7 \\
\hline 13 & estimation error & 7 \\
\hline 14 & estimation risk & 7 \\
\hline 15 & finance & 7 \\
\hline 16 & optimization & 7 \\
\hline 17 & out-of-sample performance & 7 \\
\hline 18 & risk & 7 \\
\hline 19 & sharpe ratio & 7 \\
\hline 20 & genetic algorithm & 6 \\
\hline 21 & investment analysis & 6 \\
\hline 22 & markowitz & 6 \\
\hline 23 & monte carlo simulation & 6 \\
\hline 24 & portfolio construction & 6 \\
\hline 25 & portfolio management & 6 \\
\hline 26 & cvar & 5 \\
\hline 27 & mean-variance analysis & 5 \\
\hline 28 & mean-variance model & 5 \\
\hline 29 & naive diversification & 5 \\
\hline 30 & portfolio & 5 \\
\hline
\end{tabular}

Fonte: Dados da Scopus elaborado pelo autor.

$\mathrm{Na}$ Tabela 12 acima as keywords simulation, out of sample performance, e Monte Carlo simulation aparecem, respectivamente, com a frequência de 8, 7 e 6 ocorrências, e as keywords estimation error e estimation risk aparecem ambas com a frequência de 7 ocorrências. Na classificação total das mais frequentes keywords não aparecem keywords relacionadas especificamente à modelos de otimização com simulação que apresentem a distribuição de frequência empírica dos ótimos simulados ou à métricas do risco com espaço objetivo aumentado, como evoluções do modelo de portfólio E-V eficiente.

As Tabela 13 à Tabela 18 a seguir apresentam a classificação da frequência das duzentas e cinquenta keywords mais frequentes nas publicações da pesquisa na Scopus com o segundo conjunto de palavras chave, classificadas em seis clusters: Markowitz, financial data processing, portfolio selection, economic and social effects, computer simulation e risk assessment. As keywords relacionadas com a pesquisa na Scopus com o segundo conjunto de palavras chaves apresentam uma frequência total de 52 ocorrências. As keywords relacionadas com a pesquisa na Scopus com o terceiro conjunto de palavras chaves apresentam uma frequência total de 54 ocorrências. Não aparecem keywords relacionadas especificamente à modelos de otimização com simulação que apresentem a distribuição de frequência empírica dos ótimos simulados ou à métricas do risco com espaço objetivo aumentado, como evoluções do modelo de portfólio E-V eficiente. 
Research, Society and Development, v. 10, n. 4, e57310413802, 2021

(CC BY 4.0) | ISSN 2525-3409 | DOI: http://dx.doi.org/10.33448/rsd-v10i4.13802

Tabela 13. Cluster Markowitz - Scopus, segundo conjunto de palavras chave.

\begin{tabular}{r|l|r|l}
\hline Cluster & markowitz & Cluster_Label & 1 \\
\hline Occurrences & Words & Occurrences & Words \\
\hline 8 & portfolio selection problems & 1 & c-v method \\
\hline 6 & particle swarm optimization (pso) & 1 & chance constraint \\
\hline 2 & fuzzy random variable & 1 & constraint functions \\
\hline 2 & particle swarm optimization algorithm & 1 & decision systems \\
\hline 5 & numerical experiments & 1 & fuzzy random \\
\hline 2 & solution methods & 1 & monte carlo \\
\hline 5 & problem solving & 1 & optimization criteria \\
\hline 5 & numerical methods & 1 & pso algorithms \\
\hline 3 & random variables & 1 & return level \\
\hline 3 & probability distributions & 1 & type ii \\
\hline 3 heuristic algorithms & 1 & uncertain decision systems \\
\hline 10 & markowitz & 1 & variance constraints \\
\hline 3 & stochastic programming & 1 & approximation theory \\
\hline 4 & expected return & 1 & c (programming language) \\
\hline 1 & approximation methods & & \\
\hline & & \\
\hline
\end{tabular}

Fonte: Dados da Scopus elaborado pelo autor.

Na Tabela 13 acima, cluster de Markowitz, a frequência em relação a keyword Monte Carlo é de 1 ocorrência. Não aparecem keywords relacionadas especificamente à modelos de otimização com simulação que apresentem a distribuição de frequência empírica dos ótimos simulados ou à métricas do risco com espaço objetivo aumentado, como evoluções do modelo de portfólio E-V eficiente. 
Tabela 14. Cluster Processamento de dados financeiros - Scopus, segundo conjunto de palavras chave.

\begin{tabular}{|c|c|c|c|}
\hline Cluster & financial data processing & Cluster_Label & 2 \\
\hline Occurrences & Words & Occurrences & Words \\
\hline 4 & forecasting & \begin{tabular}{r|}
13 \\
\end{tabular} & mean-variance portfolios \\
\hline 5 & investment strategy & 5 & optimization problems \\
\hline 5 & estimation & 4 & regularization \\
\hline 4 & normal distribution & 5 & investment analysis \\
\hline 5 & empirical studies & 2 & sparse portfolio \\
\hline 12 & optimal portfolios & 7 & linear programming \\
\hline 3 & earnings & 2 & operations research \\
\hline 9 & intelligent systems & 4 & portfolio choice \\
\hline 16 & monte carlo methods & 3 & sharpe ratios \\
\hline 14 & risk perception & 4 & empirical analysis \\
\hline 68 & financial data processing & 4 & investment portfolio \\
\hline 27 & mean variance & 3 & correlation matrix \\
\hline 44 & portfolio optimization & 2 & robust estimators \\
\hline 59 & investments & 3 & mean-variance portfolio optimization \\
\hline 6 & multi-period mean-variance & 2 & diversification strategies \\
\hline 4 & cvar & 3 & mean-variance analysis \\
\hline 53 & optimization & 3 & benchmark portfolios \\
\hline 9 & simulation & 2 & rebalancing \\
\hline 3 & wind power & 3 & uncertainty \\
\hline 2 & time series & 2 & uncertainty analysis \\
\hline 14 & portfolio managements & 3 & portfolio strategies \\
\hline 6 & portfolio optimisation & 2 & risks \\
\hline 3 & convex optimization & 2 & financial crisis \\
\hline 8 & estimation errors & 2 & portfolio constraints \\
\hline 4 & optimal strategies & 3 & sample sizes \\
\hline 19 & mean variance model & 3 & closed form \\
\hline 4 & optimization method & 3 & monte carlo simulation \\
\hline 12 & covariance matrix & 2 & chinese stock market \\
\hline 6 & asset allocation & 2 & making decision \\
\hline 9 & economics & 2 & optimal investments \\
\hline $3 \mathrm{~s}$ & solar energy & 3 & management science \\
\hline 4 & optimal weight & 2 & investment risks \\
\hline
\end{tabular}

Fonte: Dados da Scopus elaborado pelo autor.

$\mathrm{Na}$ Tabela 14 acima, cluster de Processamento de dados financeiros, a frequência em relação as keywords estimation, Monte Carlo methods, simulation, estimation errors, sample sizes e Monte Carlo simulation é de respectivamente 5, 16, 9, 8, 3 e 3 ocorrências. Não aparecem keywords relacionadas especificamente à modelos de otimização com simulação que apresentem a distribuição de frequência empírica dos ótimos simulados ou à métricas do risco com espaço objetivo aumentado, como evoluções do modelo de portfólio E-V eficiente. 
Research, Society and Development, v. 10, n. 4, e57310413802, 2021

(CC BY 4.0) | ISSN 2525-3409 | DOI: http://dx.doi.org/10.33448/rsd-v10i4.13802

Tabela 15. Cluster Seleção de portfólios - Scopus, segundo conjunto de palavras chave.

\begin{tabular}{r|l|l|l}
\hline Cluster & portfolio selection & Cluster_Label & 3 \\
\hline Occurrences & Words & Occurrences & Words \\
\hline 21 & financial markets & 6 & integer programming \\
\hline 3 & efficient portfolio & 6 & cardinality constraints \\
\hline 26 & portfolio selection & 9 & finance \\
\hline 3 & machine learning & optimal portfolio selection & constrained portfolios \\
\hline 7 & fuzzy sets & 8 & artificial intelligence \\
\hline 8 & portfolio selection models & 5 & entropy \\
\hline 2 & listribution functions & 5 & multi objective evolutionary algorithms \\
\hline 10 & electronic trading & 2 & statistical methods \\
\hline 12 & genetic algorithms & 2 & higher moments \\
\hline 9 & higher order statistics & 4 & combinatorial optimization \\
\hline 4 & learning systems & 2 & polynomial approximation \\
\hline 13 & multiobjective optimization & 7 & conditional value-at-risk \\
\hline 4 & lhanghai stock exchanges & 2 & lynamic programming \\
\hline 4 & lustering algorithms & 2 & sorting \\
\hline 13 & value engineering & 8 & evolutionary algorithms \\
\hline 2 & support vector machines & 2 & strength pareto evolutionary algorithm \\
\hline 2 & financial time series & 2 & value at risk \\
\hline 11 & quadratic programming & 7 & transaction cost \\
\hline 5 & fuzzy variable & 10 & algorithms \\
\hline 3 & portfolio turnover & 2 & constraint theory \\
\hline 10 & constrained optimization & 2 & investment returns \\
\hline & & \\
\hline & & \\
\hline & &
\end{tabular}

Fonte: Dados da Scopus elaborado pelo autor.

Na Tabela 15 acima, cluster de Seleção de portfólios, não aparecem keywords relacionadas especificamente à modelos de otimização com simulação que apresentem a distribuição de frequência empírica dos ótimos simulados ou à métricas do risco com espaço objetivo aumentado, como evoluções do modelo de portfólio E-V eficiente.

Tabela 16. Cluster Efeitos econômicos e sociais - Scopus, segundo conjunto de palavras chave.

\begin{tabular}{|c|c|c|c|}
\hline Cluster & economic and social effects & Cluster_Label & 4 \\
\hline Occurrences & Words & Occurrences & Words \\
\hline 7 & economic and social effects & $\mathbf{2}$ & optimal controls \\
\hline 3 & robust optimization & 1 & uncertainty quantifications \\
\hline 1 & equivalence classes & 1 & petroleum reservoir engineering \\
\hline 1 & geology & 1 & borehole logging \\
\hline 1 & oil fields & 1 & gas production \\
\hline 1 & oil shale & 1 & hydrocarbon reservoir \\
\hline 1 & oil well flooding & 1 & oil field \\
\hline 1 & optimal control systems & 1 & permeability \\
\hline 1 & petroleum reservoirs & 1 & reservoir characterization \\
\hline 1 & water injection & 1 & strategic approach \\
\hline 1 & well flooding & 1 & trade-off \\
\hline 1 & oil production & 1 & nucleopolyhedrovirus \\
\hline
\end{tabular}

Fonte: Dados da Scopus elaborado pelo autor.

Na Tabela 16 acima, cluster de Efeitos econômicos e sociais, não aparecem keywords relacionadas especificamente à modelos de otimização com simulação que apresentem a distribuição de frequência empírica dos ótimos simulados ou à métricas do risco com espaço objetivo aumentado, como evoluções do modelo de portfólio E-V eficiente. 
Research, Society and Development, v. 10, n. 4, e57310413802, 2021

(CC BY 4.0) | ISSN 2525-3409 | DOI: http://dx.doi.org/10.33448/rsd-v10i4.13802

Tabela 17. Cluster Simulação por computador - Scopus, segundo conjunto de palavras chave.

\begin{tabular}{|c|c|c|c|}
\hline Cluster & computer simulation & Cluster_Label & 5 \\
\hline Occurrences & Words & Occurrences & Words \\
\hline $\mathbf{2}$ & probability & 1 & animal welfare \\
\hline 5 & sampling & 12 & computer simulation \\
\hline 2 & biochemistry & 1 & edible plant \\
\hline 8 & decision making & 2 & financial management \\
\hline 2 & bacterial foraging optimization & $1 \mid$ & hazard assessment \\
\hline 8 & portfolio optimization models & $1 \mid$ & human \\
\hline 9 & investment & 3 & procedures \\
\hline 3 & resource allocation & 3 & theoretical model \\
\hline 4 & heuristic methods & 2 & animals \\
\hline 2 & risk & $1 \mid$ & hazard analysis and critical control points \\
\hline 5 & variance analysis & 1 & humans \\
\hline 4 & numerical model & 6 & models \\
\hline 5 & modern portfolio theories & 2 & theoretical \\
\hline 3 & genetic algorithm & 1 & plants \\
\hline 4 & article & 1 & edible \\
\hline 4 & portfolio diversification & 3 & controlled study \\
\hline 1 & estimation uncertainties & 3 & algorithm \\
\hline 1 & financial portfolio analysis & 3 & comparative study \\
\hline 1 & inspection and surveillance & 2 & economic \\
\hline 1 & phytosanitary & 2 & mathematical computing \\
\hline 1 & risk-based & 3 & mathematical model \\
\hline 1 & health risks & 2 & benchmarking \\
\hline 1 & food safety & 1 & bacterial foraging optimizations (bfo) \\
\hline 1 & sanitation & 1 & benchmark functions \\
\hline 1 & weight & 1 & genetic algorithm (gas) \\
\hline 1 & animalia & 1 & liquidity risk \\
\hline 2 & animal & & \\
\hline
\end{tabular}

Fonte: Dados da Scopus elaborado pelo autor.

Na Tabela 17 acima, cluster de Simulação por computador, a frequência em relação as keywords sampling, computer simulation, hazard assessment e estimation uncertainties é de respectivamente 5, 12, 1 e 1 ocorrências. Não aparecem keywords relacionadas especificamente à modelos de otimização com simulação que apresentem a distribuição de frequência empírica dos ótimos simulados ou à métricas do risco com espaço objetivo aumentado, como evoluções do modelo de portfólio E-V eficiente. 
Research, Society and Development, v. 10, n. 4, e57310413802, 2021

(CC BY 4.0) | ISSN 2525-3409 | DOI: http://dx.doi.org/10.33448/rsd-v10i4.13802

Tabela 18. Cluster Avaliação do risco - Scopus, segundo conjunto de palavras chave.

\begin{tabular}{|c|c|c|c|}
\hline Cluster & risk assessment & Cluster_Label & 6 \\
\hline Occurrences & Words & Occurrences & Words \\
\hline 4 & planning & \begin{tabular}{|r|}
$\mathbf{2}$ \\
\end{tabular} & computer programming \\
\hline 18 & efficient frontier & 5 & economic analysis \\
\hline 3 & global optimization & 6 & financial market \\
\hline 2 & statistical tests & 5 & mean-variance approach \\
\hline 12 & stochastic systems & 4 & risk analysis \\
\hline 7 & stochastic models & 3 & automation \\
\hline 6 & portfolio theories & 5 & sales \\
\hline 33 & commerce & 2 & distributed power generation \\
\hline 15 & costs & 4 & electricity market \\
\hline 2 & investment decisions & 5 & stock market \\
\hline 37 & risk assessment & 3 & data envelopment analysis \\
\hline 2 & financial data & 2 & electric generators \\
\hline 2 & electric industry & 2 & natural gas \\
\hline 5 & power markets & 2 & empirical evaluations \\
\hline 3 & computation theory & 2 & risk measures \\
\hline 17 & risk management & 2 & dea models \\
\hline 4 & electric utilities & 2 & site selection \\
\hline 4 & electricity & 2 & industry \\
\hline
\end{tabular}

Fonte: Dados da Scopus elaborado pelo autor.

Na Tabela 18 acima, cluster de Avaliação do risco, a frequência em relação as keywords risk assessment e risk measures é de respectivamente 37 e 2 ocorrências. Não aparecem keywords relacionadas especificamente à modelos de otimização com simulação que apresentem a distribuição de frequência empírica dos ótimos simulados ou à métricas do risco com espaço objetivo aumentado, como evoluções do modelo de portfólio E-V eficiente. 
Research, Society and Development, v. 10, n. 4, e57310413802, 2021

(CC BY 4.0) | ISSN 2525-3409 | DOI: http://dx.doi.org/10.33448/rsd-v10i4.13802

Na Figura 3 abaixo será apresentado o dendrograma das cinquenta mais frequentes keywords utilizadas na pesquisa na Scopus com o segundo conjunto de palavras chave.

Figura 3. Dendrograma das keywords - Scopus, segundo conjunto de palavras chave.

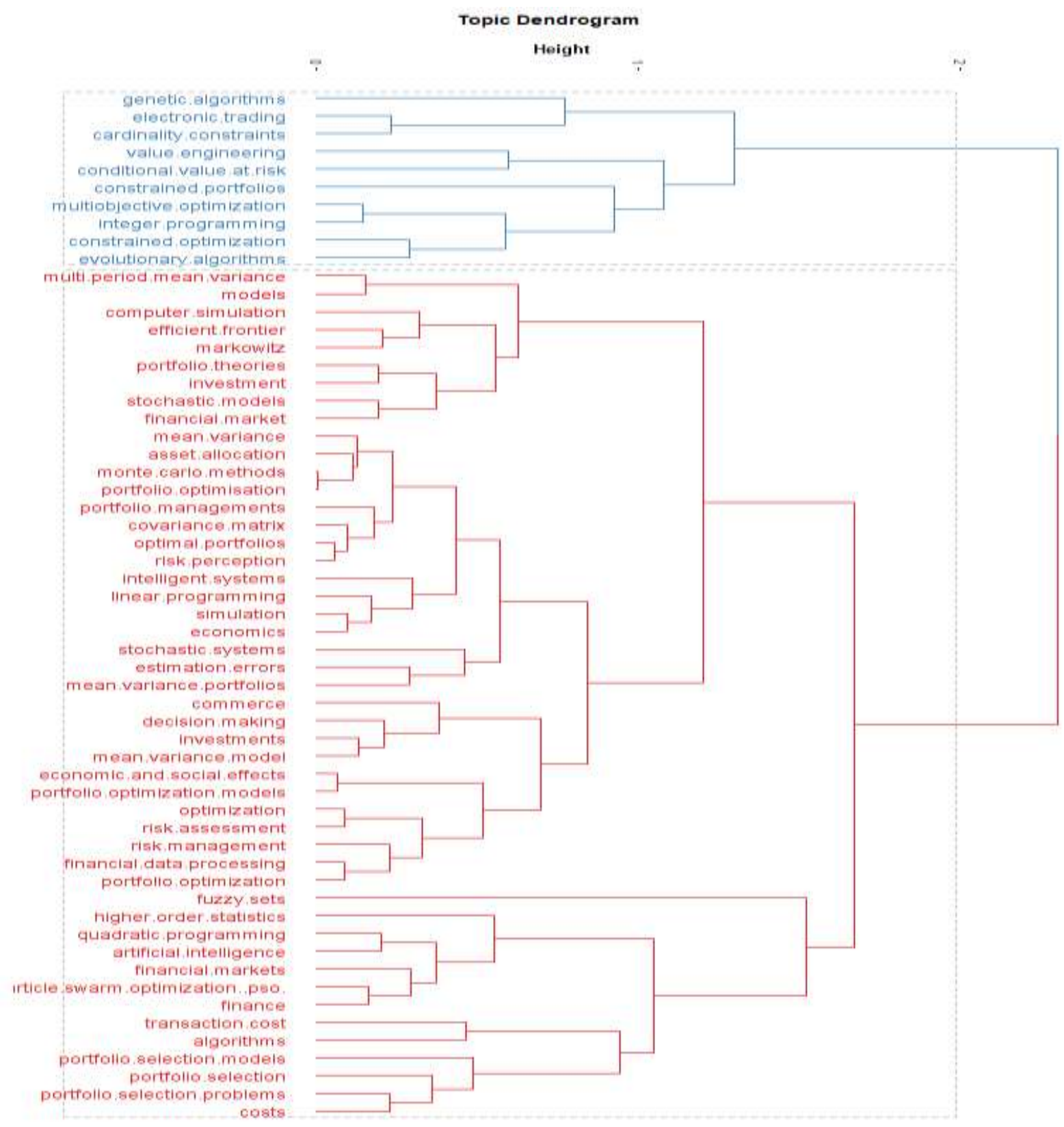

Fonte: Dados da Scopus elaborado pelo autor.

O dendrograma apresenta dois grupos específicos, no segundo grupo estão incluídas as keywords computer.simulation, Monte Carlo methods e simulation, relativas ao segundo conjunto de palavras chaves. Não aparecem keywords relacionadas especificamente à modelos de otimização com simulação que apresentem a distribuição de frequência empírica dos ótimos simulados ou à métricas do risco com espaço objetivo aumentado, como evoluções do modelo de portfólio E-V eficiente. 
Research, Society and Development, v. 10, n. 4, e57310413802, 2021

(CC BY 4.0) | ISSN 2525-3409 | DOI: http://dx.doi.org/10.33448/rsd-v10i4.13802

Na Figura 4 abaixo será apresentado o dendrograma das cinquenta mais frequentes keywords plus (mais frequentes keywords das referências) utilizadas na pesquisa na Scopus com o segundo conjunto de palavras chave.

Figura 4. Dendrograma das keywords plus - Scopus, segundo conjunto de palavras chave.

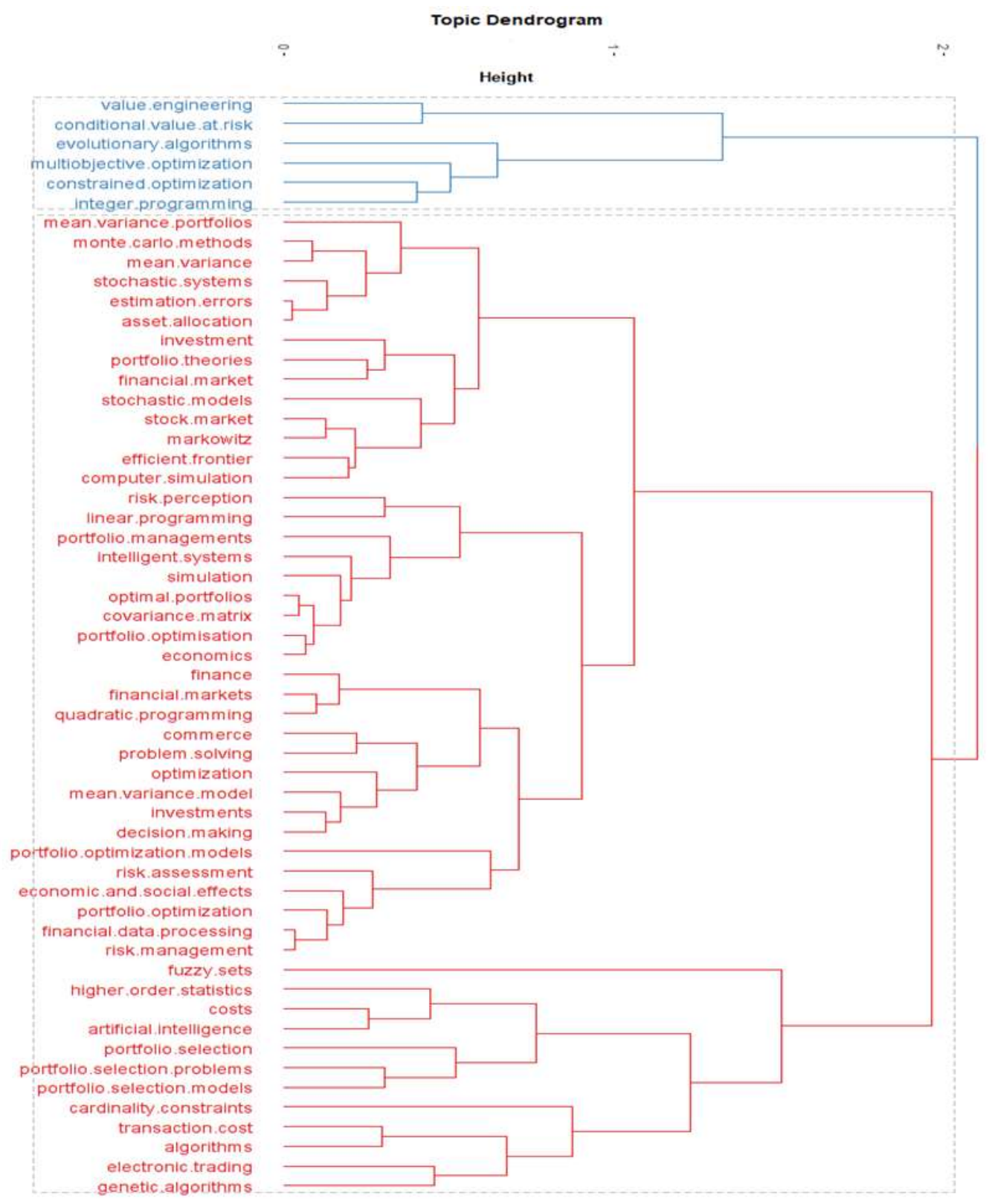

Fonte: Dados da Scopus elaborado pelo autor.

O dendrograma apresenta dois grupos específicos. No segundo grupo estão incluídas as keywords Monte.Carlo.method, computer.simulation e simulation, relativas ao segundo conjunto de palavras chaves. Não aparecem keywords relacionadas especificamente à modelos de otimização com simulação que apresentem a distribuição de frequência empírica dos ótimos simulados ou à métricas do risco com espaço objetivo aumentado, como evoluções do modelo de portfólio E-V eficiente. 
Será apresentada agora, para a pesquisa na Scopus com o segundo conjunto de palavras chave, uma nova forma de acoplamento de documentos denominada co-occurrence network que é definida como a frequência com que documentos são citados em conjunto por outro documento. A frequência de co-citação de dois artigos científicos pode ser determinada comparando a citação de documentos na base de dados da Scopus e contando entradas idênticas. A rede de co-citação pelas cinquenta principais keywords da pesquisa na Scopus com o segundo conjunto de palavras chave é apresentada abaixo na Figura 5.

Figura 5. Rede de co-citação - Scopus, segundo conjunto de palavras chave.

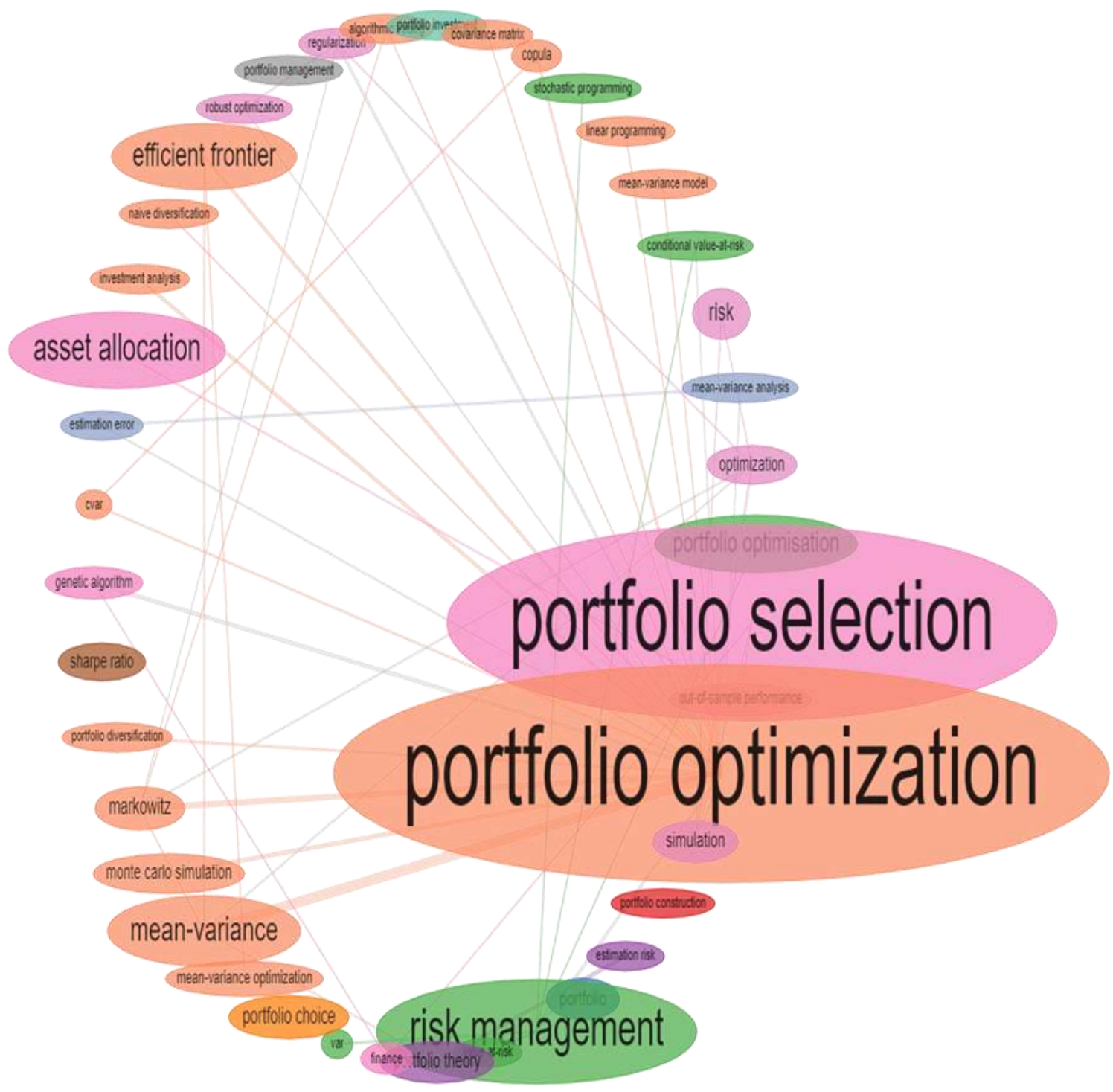

Fonte: Dados da Scopus elaborado pelo autor.

Para uma melhor visualização da rede com em relação à keyword Monte Carlo simulation vamos aplicar um filtro. 
Quando é aplicado um filtro, na rede acima, com a keyword Monte Carlo simulation, se pode verificar quais são as interrelações da keyword Monte Carlo simulation com as demais keywords, conforme a Figura 6 a seguir.

Figura 6. Rede de co-citação filtrada para keyword Monte Carlo simulation.

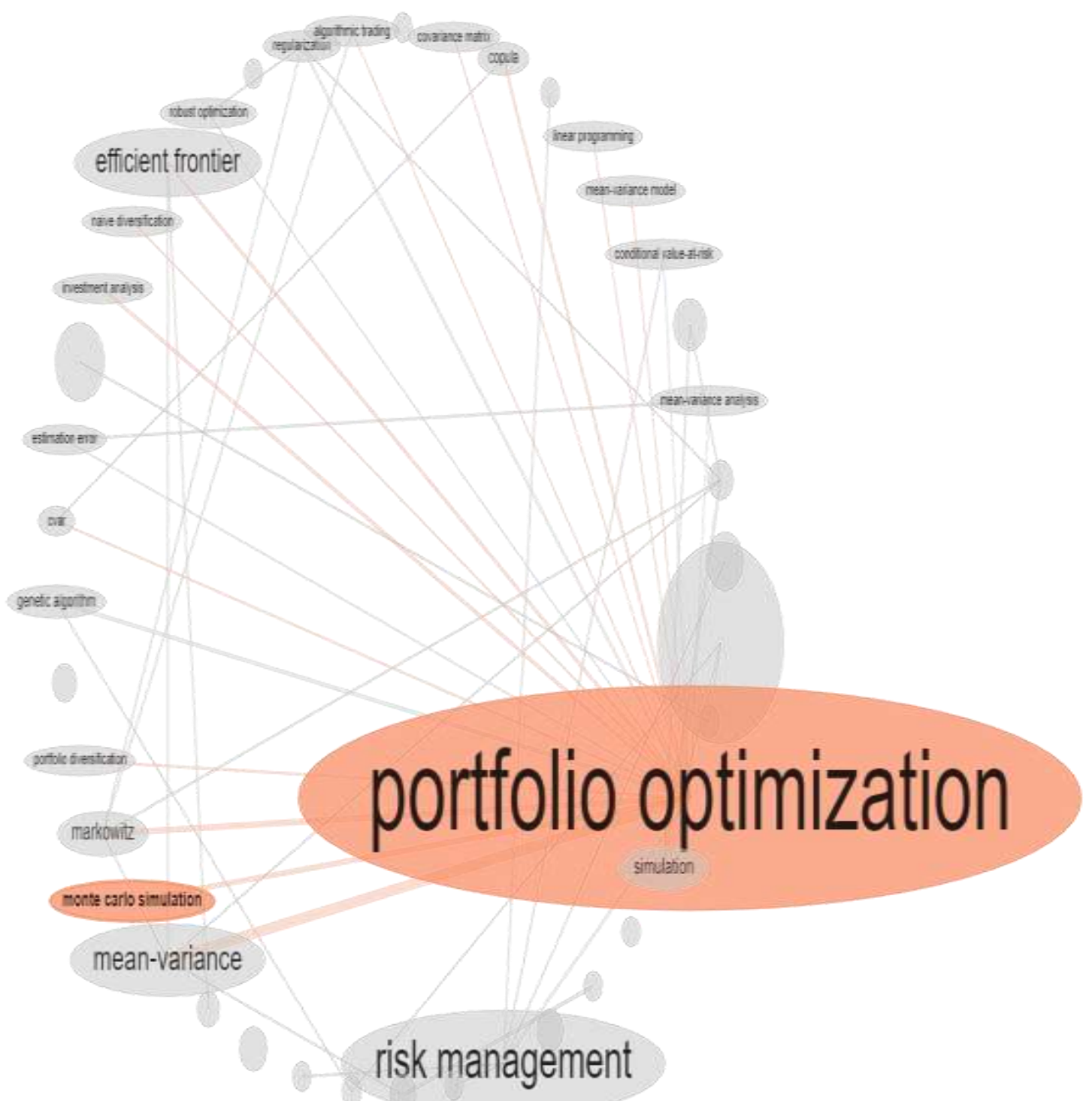

Fonte: Dados da Scopus elaborado pelo autor.

Na Figura 6 acima, as linhas em rosa identificam as keywords que tem interrelações com a keyword Monte Carlo simulation. Estas interrelações são apresentadas Tabela 19 abaixo. 
Tabela 19. Principais keywords ligadas a keyword Monte Carlo simulation.

\begin{tabular}{c}
\hline Principais keywords ligadas a keyword monte carlo simulation \\
\hline algorithmic trading \\
\hline conditional value-at-risk \\
\hline copula \\
\hline covariance matrix \\
\hline cvar \\
\hline efficient frontier \\
\hline investment analysis \\
\hline linear programming \\
markowitz \\
\hline mean-variance \\
\hline mean-variance analysis \\
\hline mean-varianve model \\
\hline naive diversification \\
\hline portfolio diversification
\end{tabular}

Fonte: Dados da Scopus elaborado pelo autor.

Percebe-se nas Figura 6 e na Tabela 19 acima que as principais interrelações da keyword Monte Carlo simulation são com keywords que dizem respeito a modelos que tratam os resultados pelo método de concentração de dados, ou seja, apresentando como resultado medidas de posição e medidas de dispersão (por exemplo média e variância). Não aparecem keywords relacionadas especificamente à modelos de otimização com simulação que apresentem a distribuição de frequência empírica dos ótimos simulados ou à métricas do risco com espaço objetivo aumentado, como evoluções do modelo de portfólio E-V eficiente. 
Dando continuidade à Segunda Etapa da apresentação bibliométrica, será apresentado a seguir os resultados da pesquisa na Scopus com o terceiro conjunto de palavras chave iniciando pelo volume de produção anual no período conforme apresentado na Gráfico 5 abaixo.

Gráfico 5. Volume de produção anual - Scopus, terceiro conjunto de palavras chave.

\section{Annual Scientific Production}

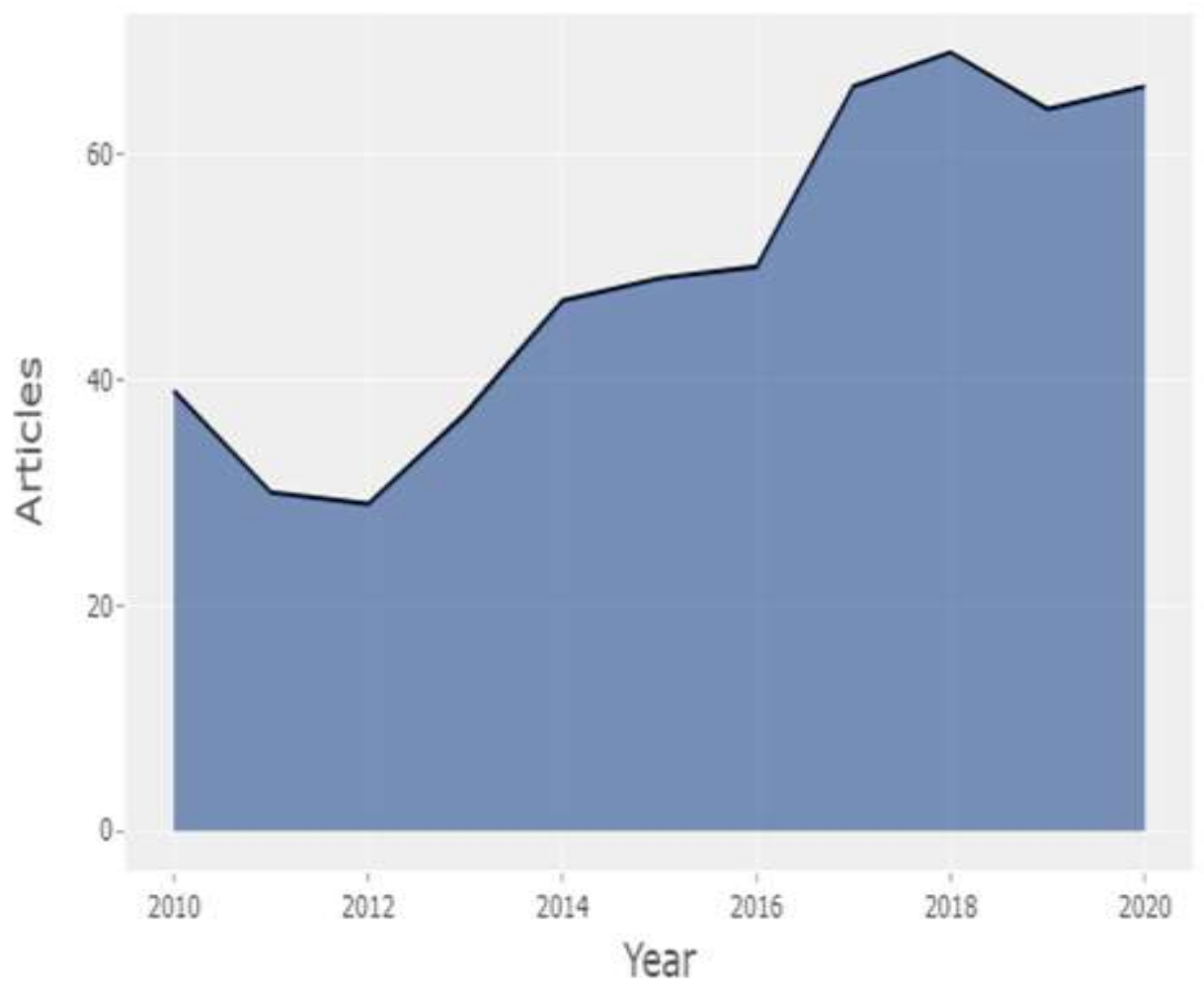

Fonte: Dados da Scopus elaborado pelo autor.

A pesquisa na Scopus com o primeiro conjunto de palavras chave é uma pesquisa generalizada sobre as extensões do modelo de portfólios E-V eficientes, inclusive métricas do risco e modelos de simulação pelo método de Monte Carlo e tem seu pico de produção em 2020 com um volume médio anual de aproximadamente cento e quarenta e cinco artigos científicos. Já a pesquisa na Scopus com o segundo conjunto de palavras chave é uma pesquisa específica sobre as extensões do modelo de portfólios E-V eficientes para modelos de simulação pelo método de Monte Carlo, tem seu pico de produção em 2017 e um volume médio anual de aproximadamente trinta e cinco artigos científicos. A pesquisa na Scopus com o terceiro conjunto de palavras chave é uma pesquisa específica sobre as extensões do modelo de portfólios E-V eficientes para métricas do risco, tem seu pico de produção em 2018 e um volume médio anual de aproximadamente cinquenta artigos científicos.

As vinte mais relevantes fontes utilizadas nas publicações da pesquisa na Scopus com o terceiro conjunto de palavras chave são apresentadas no Gráfico 6 abaixo. 
Research, Society and Development, v. 10, n. 4, e57310413802, 2021

(CC BY 4.0) | ISSN 2525-3409 | DOI: http://dx.doi.org/10.33448/rsd-v10i4.13802

Gráfico 6. Fontes mais relevantes de publicações - Scopus, terceiro conjunto de palavras chave.

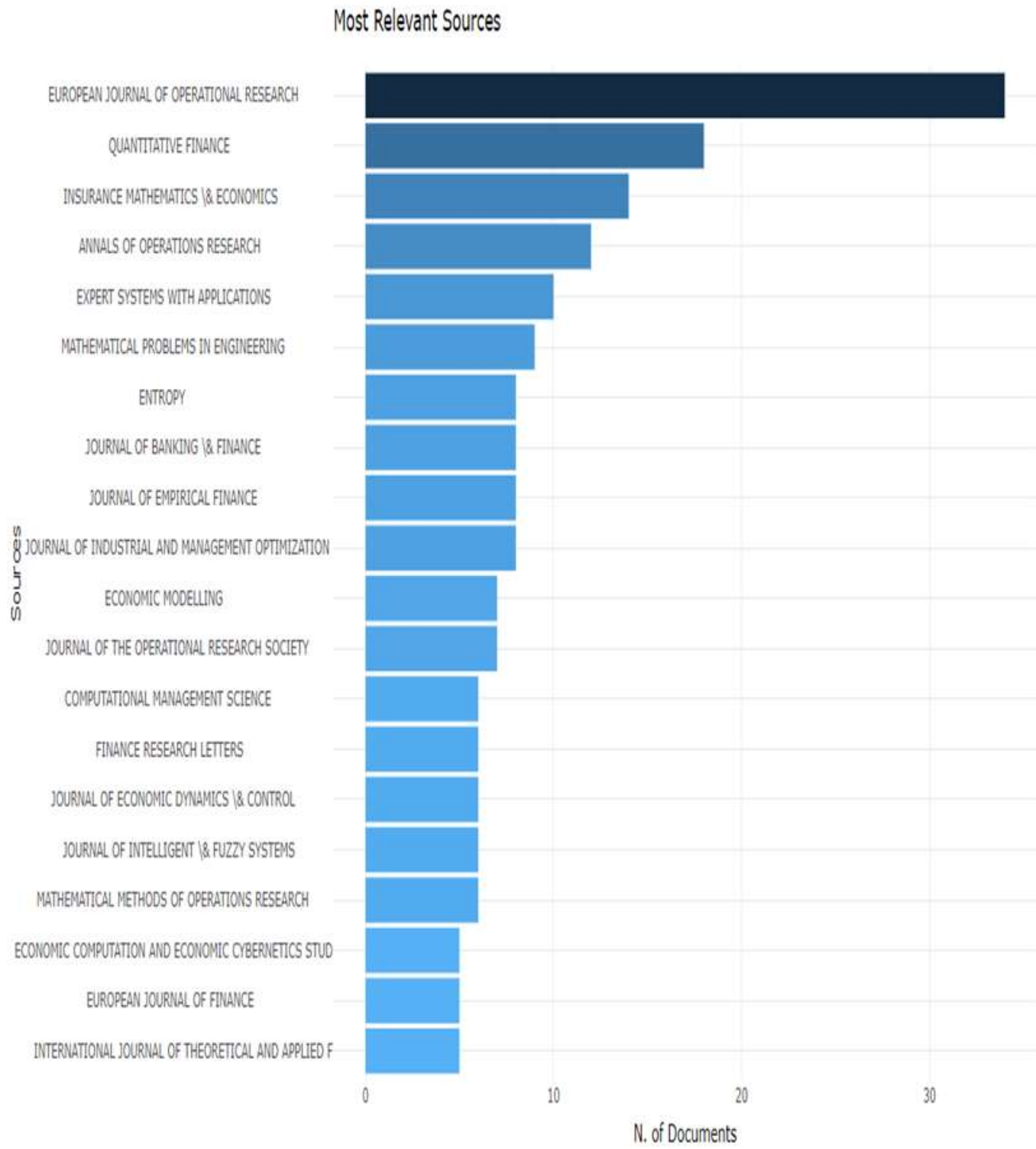

Fonte: Dados da Scopus elaborado pelo autor.

No Gráfico 6 acima podemos perceber que as fontes mais relevantes da pesquisa na Scopus com o terceiro conjunto de palavras chaves são o European Journal of Operational Research, o Quantitative Finance e o Insurance Mathematics $1 \&$ Economics.

A Tabela 20 abaixo apresenta a classificação das trinta mais frequentes keywords utilizadas nas publicações da pesquisa na Scopus com o terceiro conjunto de palavras chave. 
Tabela 20. Keywords mais frequentes - Scopus, terceiro conjunto de palavras chave.

\begin{tabular}{|c|c|c|}
\hline & Words & Occurrences \\
\hline 1 & portfolio optimization & 85 \\
\hline 2 & portfolio selection & 64 \\
\hline 3 & portfolio & 40 \\
\hline 4 & mean-variance & 34 \\
\hline 5 & optimization & 30 \\
\hline 6 & risk management & 20 \\
\hline 7 & risk & 19 \\
\hline 8 & efficient frontier & 17 \\
\hline 9 & asset allocation & 16 \\
\hline 10 & conditional value-at-risk & 16 \\
\hline 11 & value-at-risk & 15 \\
\hline 12 & finance & 14 \\
\hline 13 & mean-variance analysis & 14 \\
\hline 14 & mean-variance model & 14 \\
\hline 15 & mean-variance optimization & 14 \\
\hline 16 & selection & 13 \\
\hline 17 & entropy & 12 \\
\hline 18 & estimation risk & 12 \\
\hline 19 & g11 & 12 \\
\hline 20 & risk measure & 12 \\
\hline 21 & uncertain variable & 12 \\
\hline 22 & programming & 11 \\
\hline 23 & cvar & 10 \\
\hline 24 & portfolio choice & 10 \\
\hline 25 & portfolio optimisation & 10 \\
\hline 26 & portfolio theory & 10 \\
\hline 27 & sharpe ratio & 10 \\
\hline 28 & downside risk & 9 \\
\hline 29 & model & 9 \\
\hline 30 & portfolio management & 9 \\
\hline
\end{tabular}

Fonte: Dados da Scopus elaborado pelo autor.

Na Tabela 20 acima as keywords conditional value-at-risk, value-at-risk, estimation risk, risk measure e cvar aparecem, respectivamente, com a frequência de 10,11,18, 20 e 23 referências, e a frequência total em relação as keywords associadas com assessment, metrics, measures e estimation é de 161 referências. Na classificação total das mais frequentes keywords não aparecem keywords relacionadas especificamente à modelos de otimização com simulação que apresentem a distribuição de frequência empírica dos ótimos simulados ou à métricas do risco com espaço objetivo aumentado, como evoluções do modelo de portfólio E-V eficiente.

As Tabela 21 à Tabela 28 a seguir apresentam a classificação da frequência das duzentas e cinquenta keywords mais frequentes nas publicações da pesquisa na Scopus com o terceiro conjunto de palavras chave classificadas em oito clusters: portfolio otimization, portfolio, estimation, asset allocation, efficient frontier, martingale, uncertainty e portfolio theory. As keywords relacionadas com a pesquisa na Scopus com o terceiro conjunto de palavras chaves apresentam uma frequência total de 72 ocorrências. As keywords relacionadas com a pesquisa na Scopus com o segundo conjunto de palavras chaves apresentam uma frequência total de 20 ocorrências. 
Research, Society and Development, v. 10, n. 4, e57310413802, 2021

(CC BY 4.0) | ISSN 2525-3409 | DOI: http://dx.doi.org/10.33448/rsd-v10i4.13802

Tabela 21. Cluster otimização de portfólio - Scopus, terceiro conjunto de palavras chave.

\begin{tabular}{|c|c|c|c|}
\hline Cluster & portfolio otimization & Cluster_Label & 1 \\
\hline Occurrences & Words & Occurrences & Words \\
\hline 64 & portfolio selection & 3 & elliptical distributions \\
\hline 4 & cardinality & 2 & principle \\
\hline 85 & portfolio optimization & 3 & constraints \\
\hline 6 & regularization & 2 & systematic risk \\
\hline 2 & 1 & 11 & programming \\
\hline 14 & mean-variance optimization & 5 & copula \\
\hline 4 & possibility theory & 3 & semi-variance \\
\hline 5 & genetic algorithm & 4 & evolutionary algorithms \\
\hline 34 & mean-variance & 12 & g11 \\
\hline 3 & particle swarm optimization & 4 & deviation \\
\hline 2 & information entropy & 6 & dynamic \\
\hline 9 & risk measures & 4 & sparse portfolio \\
\hline 3 & machine learning & 3 & minimum variance portfolio \\
\hline 8 & mean-variance portfolio & 2 & markowitz model \\
\hline 3 & var & 5 & linear programming \\
\hline 10 & portfolio optimisation & 6 & markowitz \\
\hline 20 & risk management & 2 & dynamic conditional \\
\hline 4 & optimal control & 6 & dynamic programming \\
\hline 12 & uncertain variable & 4 & multiperiod portfolio selection \\
\hline 6 & measure & 7 & credibility measure \\
\hline 4 & factor model & 3 & mean-variance theory \\
\hline 3 & shrinkage & 4 & transaction cost \\
\hline 3 & lasso & 4 & robustness \\
\hline 12 & risk measure & 14 & mean-variance analysis \\
\hline 10 & cvar & 12 & entropy \\
\hline 14 & finance & 7 & multi-period portfolio selection \\
\hline 5 & black-litterman model & 3 & genetic \\
\hline 4 & cardinality constraints & 2 & decision analysis \\
\hline 7 & parameter uncertainty & 3 & multiobjective optimization \\
\hline 4 & maximum principle & 4 & short selling \\
\hline 2 & ambiguity & 3 & energy \\
\hline 3 & time consistency & 3 & naive diversification \\
\hline 3 & hamilton-jacobi-bellman equation & 3 & robust statistics \\
\hline 8 & investment analysis & 4 & time inconsistency \\
\hline 2 & spectral risk measure & 4 & uncertainty theory \\
\hline 3 & black-litterman & 4 & c61 \\
\hline 5 & expected shortfall & 3 & stochastic \\
\hline 2 & median & 3 & bayes-stein \\
\hline 3 & robust portfolio & 3 & econometrics \\
\hline 3 & risk assessment & 3 & performance \\
\hline 8 & robust optimization & 2 & c14 \\
\hline 3 & statistics & 3 & mean-variance portfolio selection \\
\hline 5 & out-of-sample & 4 & optimal portfolio \\
\hline 16 & conditional value-at-risk & 4 & tail variance \\
\hline 3 & optimal & 4 & utility functions \\
\hline 6 & multi-objective & 4 & conditional value at risk \\
\hline 4 & algorithm & 3 & efficiency \\
\hline 2 & maximum drawdown & 3 & uncertainty modeling \\
\hline 3 & regime switching & 2 & c1 \\
\hline 8 & estimation error & 4 & capm \\
\hline 3 & portfolio performance & & \\
\hline
\end{tabular}

Fonte: Dados da Scopus elaborado pelo autor.

Na Tabela 21 acima, cluster de otimização de portfólio, a frequência em relação as keywords risk measures, measure, risk measure, spectral risk measure, risk assessment, estimation error e credibility measure é de respectivamente 9, 6, 12, 2, 3, 8 e 7 ocorrências e a frequência em relação a keyword out-of-sample é de 5 ocorrências. Não aparecem keywords relacionadas especificamente à modelos de otimização com simulação que apresentem a distribuição de frequência empírica dos ótimos simulados ou à métricas do risco com espaço objetivo aumentado, como evoluções do modelo de portfólio E-V eficiente. 
Tabela 22. Cluster portfólio - Scopus, terceiro conjunto de palavras chave.

\begin{tabular}{r|l|l|l}
\hline Cluster & portfolio & Cluster_Label & 2 \\
\hline Occurrences & Words & Occurrences & Words \\
\hline 40 & portfolio & 4 & value at risk \\
\hline 9 & portfolio management & 3 & investment risk \\
\hline 3 & analysis & 9 & downside risk \\
\hline 2 & fuzzy sets & 8 & semivariance \\
\hline 12 & estimation risk & 6 & skewness \\
\hline 3 & genetic algorithms & 6 forecasting \\
\hline 2 & asset selection & 4 & rebalancing \\
\hline 19 & risk & 8 & variance \\
\hline 7 & management & 2 & decision-making \\
\hline 14 & value-at-risk & 9 & transaction costs \\
\hline 13 & selection & 4 & fuzzy random variable \\
\hline 3 & liquidity risk & 4 & conditional \\
\hline 30 & optimization & 4 & efficient frontiers \\
\hline 2 & algorithms & stability \\
\hline 4 & risk analysis & convex optimization \\
\hline 4 & out-of-sample performance & \\
\hline
\end{tabular}

Fonte: Dados da Scopus elaborado pelo autor.

$\mathrm{Na}$ Tabela 22 acima, cluster de portfólio, a frequência em relação a keyword estimation risk é de 12 ocorrências e a frequência em relação que a keyword out-of-sample performance é de 4 ocorrências. Não aparecem keywords relacionadas especificamente à modelos de otimização com simulação que apresentem a distribuição de frequência empírica dos ótimos simulados ou à métricas do risco com espaço objetivo aumentado, como evoluções do modelo de portfólio E-V eficiente.

Tabela 23. Cluster estimação - Scopus, terceiro conjunto de palavras chave.

\begin{tabular}{|c|c|c|c|}
\hline Cluster & estimation & Cluster_Label & 3 \\
\hline Occurrences & Words & Occurrences & Words \\
\hline & diversification & 2 & market efficiency \\
\hline & estimation & 2 & equity premium puzzle \\
\hline & behavioral finance & 2 & bubbles \\
\hline & applications & 6 & stochastic dominance \\
\hline & models & 4 & measures \\
\hline & econometric and statistical models & 2 & performance measures \\
\hline & covariance & 4 & cointegration \\
\hline & utility & & robust \\
\hline
\end{tabular}

Fonte: Dados da Scopus elaborado pelo autor.

Na Tabela 23 acima, cluster de estimação, a frequência em relação as keywords estimation, measures e performance measures é de respectivamente de 7, 4 e 2 ocorrências. Não aparecem keywords relacionadas especificamente à modelos de otimização com simulação que apresentem a distribuição de frequência empírica dos ótimos simulados ou à métricas do risco com espaço objetivo aumentado, como evoluções do modelo de portfólio E-V eficiente. 
Research, Society and Development, v. 10, n. 4, e57310413802, 2021

(CC BY 4.0) | ISSN 2525-3409 | DOI: http://dx.doi.org/10.33448/rsd-v10i4.13802

Tabela 24. Cluster alocação de ativos - Scopus, terceiro conjunto de palavras chave.

\begin{tabular}{r|l|l|l}
\hline Cluster & asset allocation & Cluster_Label & 4 \\
\hline Occurrences & Words & Occurrences & Words \\
\hline 16 & asset allocation & 2 & covariance matrices \\
\hline 4 & bootstrap & 2 & framework \\
\hline 4 & portfolio construction & 9 & model \\
\hline 3 & interval & 3 & data envelopment analysis \\
\hline 4 & garch & 3 & frontier \\
\hline 8 & portfolios & 4 & mutual funds \\
\hline 2 & asset pricing models & 4 & risk aversion \\
\hline 5 & performance evaluation & 10 & portfolio choice \\
\hline 5 & distribution & 2 & hedge funds \\
\hline 2 & maximum diversification & 3 & climate change \\
\hline 3 & minimum variance & 4 & portfolio analysis \\
\hline 14 & mean-variance model & 5 & shortage function \\
\hline 2 & resource management & 2 & test \\
\hline 2 & clustering algorithms & 2 & utility maximization \\
\hline
\end{tabular}

Fonte: Dados da Scopus elaborado pelo autor.

Na Tabela 24 acima, cluster alocação de ativos, não aparecem keywords relacionadas especificamente à modelos de otimização com simulação que apresentem a distribuição de frequência empírica dos ótimos simulados ou à métricas do risco com espaço objetivo aumentado, como evoluções do modelo de portfólio E-V eficiente.

Tabela 25. Cluster fronteira eficiente - Scopus, terceiro conjunto de palavras chave.

\begin{tabular}{r|l|l|l}
\hline Cluster & efficient frontier & Cluster_Label & 5 \\
\hline Occurrences & Words & Occurrences & Words \\
\hline 17 & efficient frontier & 10 & sharpe ratio \\
\hline 5 & optimal portfolio selection & 2 & covariance matrix \\
\hline 2 & equation & regression \\
\hline 4 & monte carlo simulation & theory \\
\hline 3 & expected utility maximization & shrinkage estimator \\
\hline
\end{tabular}

Fonte: Dados da Scopus elaborado pelo autor.

$\mathrm{Na}$ Tabela 25 acima, cluster de fronteira eficiente, a frequência em relação a keyword Monte Carlo simulation é de 4 ocorrências. Não aparecem keywords relacionadas especificamente à modelos de otimização com simulação que apresentem a distribuição de frequência empírica dos ótimos simulados ou à métricas do risco com espaço objetivo aumentado, como evoluções do modelo de portfólio E-V eficiente. 
Tabela 26. Cluster martingale - Scopus, terceiro conjunto de palavras chave.

\begin{tabular}{r|l|r|l}
\hline Cluster & martingale & Cluster_Label & 6 \\
\hline Occurrences & Words & Occurrences & Words \\
\hline 4 & martingale & 3 & dynamic optimality \\
\hline 3 & geometric brownian motion & 2 markov process \\
\hline 2 & mean variance analysis & \\
\hline
\end{tabular}

Fonte: Dados da Scopus elaborado pelo autor.

Na Tabela 26 acima, cluster martingale, não aparecem keywords relacionadas especificamente à modelos de otimização com simulação que apresentem a distribuição de frequência empírica dos ótimos simulados ou à métricas do risco com espaço objetivo aumentado, como evoluções do modelo de portfólio E-V eficiente.

Tabela 27. Cluster incerteza - Scopus, terceiro conjunto de palavras chave.

\begin{tabular}{r|l|r|l}
\hline Cluster & uncertainty & Cluster_Label & 7 \\
\hline Occurrences & Words & Occurrences & Words \\
\hline 9 & uncertainty & 5 & simulation \\
\hline 2 & continuous-time markowitz problem & 2 & real \\
\hline 4 & mean-variance portfolio optimization & 2 & monte carlo \\
\hline
\end{tabular}

Fonte: Dados da Scopus elaborado pelo autor.

Na Tabela 27 acima, cluster de fronteira eficiente, a frequência em relação as keywords simulation e Monte Carlo é de respectivamente 5 e 2 ocorrências. Não aparecem keywords relacionadas especificamente à modelos de otimização com simulação que apresentem a distribuição de frequência empírica dos ótimos simulados ou à métricas do risco com espaço objetivo aumentado, como evoluções do modelo de portfólio E-V eficiente.

Tabela 28. Cluster teoria de portfólio - Scopus, terceiro conjunto de palavras chave.

\begin{tabular}{l|l|l|l}
\hline Cluster & portfolio theory & Cluster_Label 8 \\
\hline Occurrences & Words & Occurrences & Words \\
\hline 10 portfolio theory & \multicolumn{2}{|c}{3 time-consistent strategy } \\
\hline 8 investment & & \\
\hline
\end{tabular}

Fonte: Dados da Scopus elaborado pelo autor.

Na Tabela 28 acima, cluster teoria de portfólio, não aparecem keywords relacionadas especificamente à modelos de otimização com simulação que apresentem a distribuição de frequência empírica dos ótimos simulados ou à métricas do risco com espaço objetivo aumentado, como evoluções do modelo de portfólio E-V eficiente. 
Research, Society and Development, v. 10, n. 4, e57310413802, 2021

(CC BY 4.0) | ISSN 2525-3409 | DOI: http://dx.doi.org/10.33448/rsd-v10i4.13802

Na Figura 7 a seguir será apresentado o dendrograma das 50 mais frequentes keywords utilizadas na pesquisa na Scopus com o terceiro conjunto de palavras chave.

Figura 7. Dendrograma das keywords - Scopus, terceiro conjunto de palavras chave.

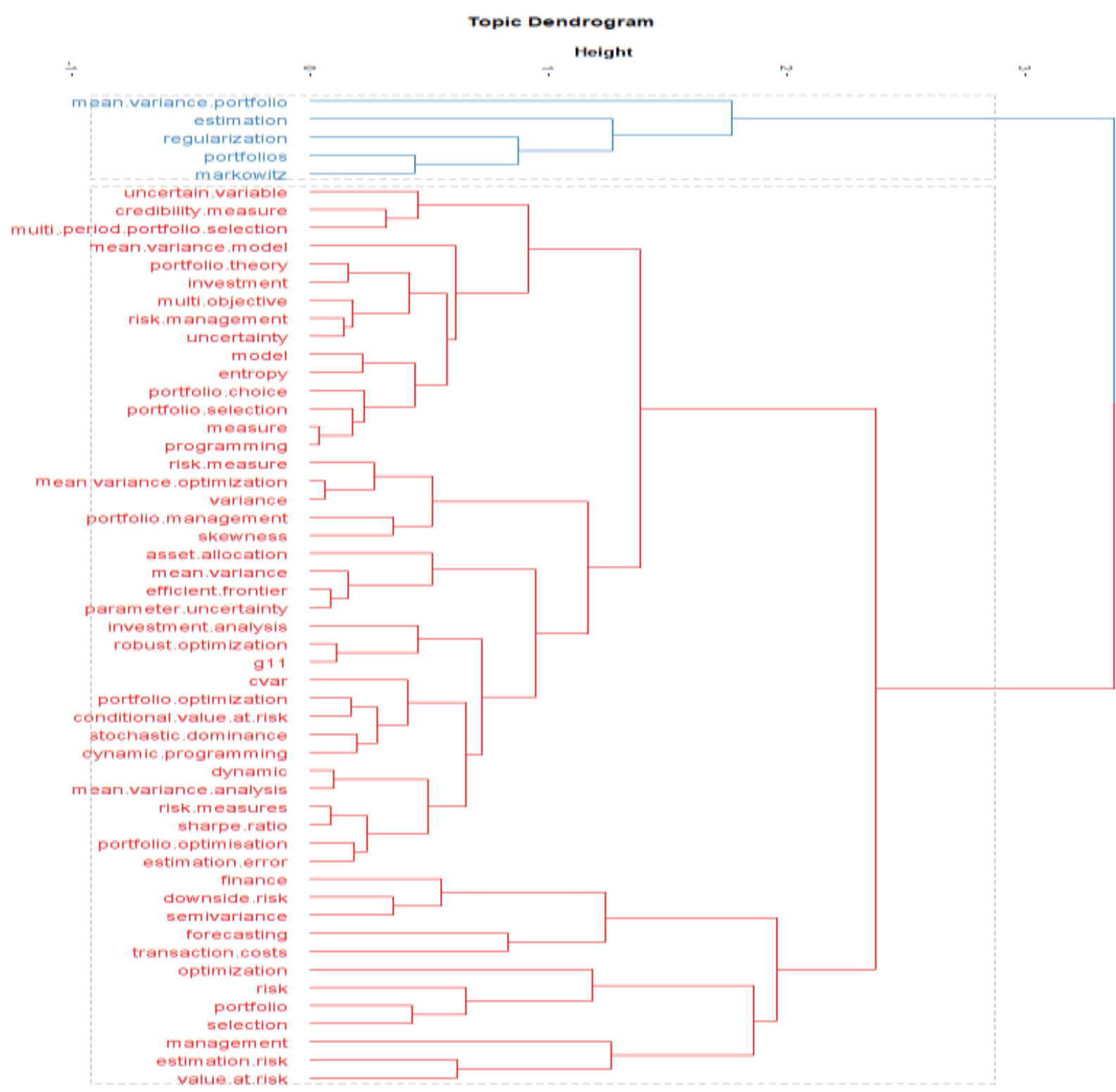

Fonte: Dados da Scopus elaborado pelo autor.

O dendrograma apresenta dois grupos específicos. No primeiro grupo está incluída a keyword estimation, relativa ao segundo conjunto de palavras chaves. No segundo grupo estão incluídas as keywords credibility.measure, measure, risk.measure, cvar, conditional.value.at.risk, risk.measures, estimation.error, downside.risk, estimation.risk e value.at.risk, relativas ao terceiro conjunto de palavras chaves. Não aparecem keywords relacionadas especificamente à modelos de otimização com simulação que apresentem a distribuição de frequência empírica dos ótimos simulados ou à métricas do risco com espaço objetivo aumentado, como evoluções do modelo de portfólio E-V eficiente. 
Na Figura 8 abaixo será apresentado o dendrograma das 50 mais frequentes keywords plus (mais frequentes keywords das referências) utilizadas na pesquisa na Scopus com o terceiro conjunto de palavras chave.

Figura 8. Dendrograma das keywords plus - Scopus, terceiro conjunto de palavras chave.

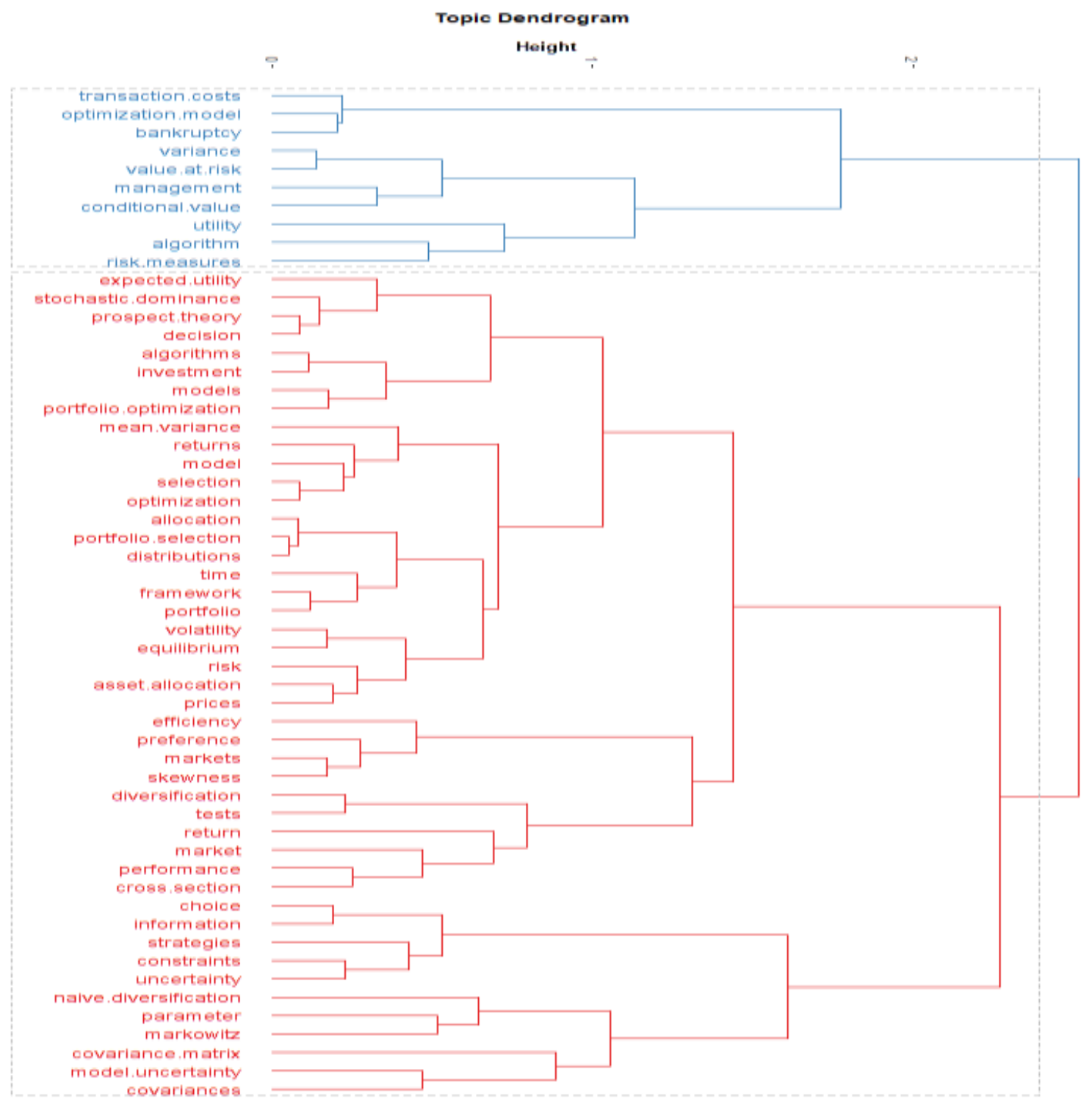

Fonte: Dados da Scopus elaborado pelo autor.

O dendrograma apresenta dois grupos específicos, mas em nenhum dos grupos aparecem palavras chaves relacionadas com o segundo ou com o terceiro conjunto de palavras chaves. Na Figura 8 as keywords das referências não apresentam keywords relacionadas diretamente com métricas do risco, indicando que a maior parte dos títulos não deve ser especificamente sobre métricas do risco. Não aparecem keywords relacionadas especificamente à modelos de otimização com simulação que apresentem a distribuição de frequência empírica dos ótimos simulados ou à métricas do risco com espaço objetivo aumentado, como evoluções do modelo de portfólio E-V eficiente. 
Na pesquisa na Scopus com o primeiro, segundo e terceiro conjunto de palavras chaves, a fonte mais relevante é o European Journal of Operational Research com 59 artigos, a fonte de referências mais citada é o Journal of Finance com 1252 citações; os três mais relevantes autores são $\mathrm{Li}, \mathrm{Li}$, e Li com respectivamente 30, 24 e 22 artigos; os autores mais citados são Markowitz, Li, e Zhou, com respectivamente 1007, 824 e 673 citações; as três mais relevantes entidades são a Sun Yat-Sen University, a Chinese University of Hong Kong e a Shangai University of Finance and Economics com respectivamente 26, 23 e 23 artigos; os três países com maior produção científica na área em questão são a China, os Estados Unidos e a Inglaterra com respectivamente 758, 314 e 153 artigos; os três países mais citados são a China, os Estados Unidos e Hong Kong com respectivamente 1528, 1354 e 531 artigos citados. As principais colaborações do Brasil são com Portugal, Espanha e Alemanha.

\section{Terceira Etapa - Filtro das pesquisas na Scopus e na Web of Science por campos específicos.}

Para confirmar o que foi identificado principalmente nas Figura 6, na Tabela 19 e na Figura 8 acima, será realizada agora uma análise e listagem dos resultados das seis pesquisas realizadas, três na base de dados da Scopus e três na base de dados da Web of Science, utilizando filtros, com dois objetivos:

- Primeiro separar por cluster de títulos específicos os documentos das bases de dados filtrados nos campos de keyword, keyword plus, abstract e title, pelas palavras chaves sampled, sampling, resampled, Monte Carlo, sample e simulation de forma que se possa concluir se existem artigos que apresentem os seus resultados na forma da distribuição de probabilidade empírica de todos os resultados ótimos de um modelo de otimização com simulação, e não pelo usual método de concentração de dados (medidas de posição e de dispersão como a média e a variância), e de que forma estes resultados seriam tratados nos documentos em questão, caso existam. As bases de dados serão analisadas, com a exclusão de títulos repetidos na mesma base ou em bases diferentes, e os títulos resultantes da análise serão classificados qualitativamente em clusters e apresentados em uma tabela.

- Segundo separar por cluster de títulos específicos os documentos das bases de dados filtrados nos campos de keyword, keyword plus, abstract e title, pelas palavras chaves assessment, metrics, measures e estimation, de forma que se possa verificar se existem artigos que apresentem seus resultados a respeito de métrica do risco na forma de uma métrica do risco com espaço objetivo aumentado, de forma a considerar um maior número de parâmetros a serem otimizados, possibilitando melhores resultados, e caso existam como são suas formulações. As bases de dados serão analisadas, com a exclusão de títulos repetidos na mesma base ou em bases diferentes, e os títulos resultantes da análise serão classificados qualitativamente em clusters e apresentados em uma tabela.

Nas pesquisas na Scopus e na Web of Science utilizando os três conjuntos de palavras chave, em um total de seis pesquisas, após a aplicação dos filtros nos campos de keyword, keyword plus, abstract e title, e exclusão dos títulos repetidos, foram selecionados cento e setenta e dois títulos filtrados pelas palavras chaves sampled, sampling, resampled, Monte Carlo, sample e simulation, para serem classificados em clusters. A Tabela 29 a abaixo apresenta o resultado do cluster de títulos para modelos específicos a respeito de simulação e a respeito do método de Monte Carlo com um total de 172 títulos.

Nas pesquisas na Scopus e na Web of Science utilizando os três conjuntos de palavras chave, em um total de seis pesquisas, após a aplicação dos filtros nos campos de keyword, keyword plus, abstract e title, e exclusão dos títulos repetidos, foram selecionados seiscentos e dez títulos filtrados pelas palavras chaves assessment, metrics, measures e estimation, para serem classificados em clusters. A Tabela 30 a seguir apresenta o resultado do cluster de títulos para métodos específicos a respeito de métricas do risco com um total de 610 títulos. 
Tabela 29. Cluster de títulos a respeito de métodos de otimização com simulação.

\begin{tabular}{|c|c|}
\hline Cluster de métodos & Número artigos \\
\hline Artificial Intelligence & 37 \\
\hline Bayes & 4 \\
\hline Bootstrap & 2 \\
\hline Cluster Analysis & 4 \\
\hline Copula & 9 \\
\hline Correlation Matrix & 5 \\
\hline Conditional Value at Risk & 3 \\
\hline Data Envelopment Analysis & 2 \\
\hline Dimension Reduction & 1 \\
\hline Diversos & 9 \\
\hline Dynamic Model & 4 \\
\hline Global Minimal Variance & 1 \\
\hline Gross-Exposure & 2 \\
\hline Importance Sample & 1 \\
\hline Kernel Density Estimator & 1 \\
\hline Linear Programming & 2 \\
\hline Model Predictive Control & 2 \\
\hline Multiperiod Model & 4 \\
\hline Operational Research & 3 \\
\hline Operational Risk & 2 \\
\hline Portfolio Analysis & 11 \\
\hline Regression & 2 \\
\hline Replica Analysis & 2 \\
\hline Robust Optimation & 5 \\
\hline Sampling Resampling Monte Carlo & 10 \\
\hline Shrinkage & 3 \\
\hline Social & 1 \\
\hline Stochastic Models & 20 \\
\hline Utilities & 19 \\
\hline Value-at-Risk & 1 \\
\hline Soma & 172 \\
\hline
\end{tabular}

Fonte: Dados da Scopus e da Web of Science elaborada pelo autor. 
Tabela 30. Cluster de títulos a respeito de métricas do risco.

\begin{tabular}{|c|c|}
\hline Cluster de métricas do risco & Número artigos \\
\hline Adaptive models & 2 \\
\hline Bayes & 7 \\
\hline Black-Litterman & 11 \\
\hline Bootstrap & 5 \\
\hline Cluster models & 3 \\
\hline Conditional value-at-risk & 9 \\
\hline Copula models & 7 \\
\hline Data envelopment analysis & 2 \\
\hline Diversification & 29 \\
\hline Diversos & 126 \\
\hline Downside models & 7 \\
\hline Dynamic optimization & 13 \\
\hline Forecast & 9 \\
\hline Foreign studies & 4 \\
\hline Fuzzy models & 31 \\
\hline Garch models & 2 \\
\hline Genetic Algorithm & 2 \\
\hline Geometric Brownian & 1 \\
\hline Interval selection & 1 \\
\hline Learning models & 7 \\
\hline Linear models & 11 \\
\hline Mean models & 37 \\
\hline Multi-objetive & 9 \\
\hline Multi-period models & 7 \\
\hline Multicriteria models & 1 \\
\hline Multivariate models & 5 \\
\hline Nonparametric & 5 \\
\hline Performance measures & 1 \\
\hline Robust optimization & 37 \\
\hline Sharpe ratio & 1 \\
\hline Shrinkage & 4 \\
\hline Stochastic models & 11 \\
\hline Tail dependence & 1 \\
\hline Value-at-risk & 7 \\
\hline Variance & 193 \\
\hline Vast Portfólio & 2 \\
\hline Soma & 610 \\
\hline
\end{tabular}

Fonte: Dados da Scopus e da Web of Science elaborada pelo autor. 
Em função da impossibilidade de listar o grande número de artigos relacionados nas Tabela 29 e Tabela 30 serão listados a seguir, para cada cluster de cada tabela, somente uma referência de cada cluster, sendo priorizada a referência mais relevante e/ou mais atual do cluster identificado. A totalidade das referências pode ser obtida pela realização das pesquisas na Scopus e na Web of Science com o conjunto de palavras chaves das pesquisas e os campos utilizados como filtro na geração das tabelas.

Assim na Tabela 29 as referências são:

- $\quad$ Artificial Intelligence: Yan, Hu, and Lai. (2018).

- $\quad$ Bayesian Models: Platanakis, Sutcliffe, and Ye. (2020).

- $\quad$ Bootstrap: Shen. et al. (2019).

- Cluster Analysis: Begusiae, and Kostanjèar. (2020).

- Copula: Chakkalakal, Hommel, and Li. (2018).

- $\quad$ Correlation Matrix: Münnix, Schäfer, and Grothe. (2014).

- $\quad$ Conditional Value at Risk: Naccarato, and Pierini. (2014).

- Data Envelopment Analysis: Xiao, Ren, and Ren. (2020).

- Dimension Reduction: Resta. (2012).

- Diversos: Jiang, Hong, and Nelson. (2020).

- $\quad$ Dynamic Model: Cong. and Oosterlee. (2016).

- $\quad$ Global Minimal Variance: Vasyl Golosnoy,et al. (2020).

- Gross-Exposure: Fan, Zhang, and Yu,(2012).

- Importance Sample: Ekblom, and Blomvall(2020).

- $\quad$ Kernel Density Estimator: Yua,et al. (2020).

- $\quad$ Linear Programming: Huang, and Lin. (2018).

- $\quad$ Model Predictive Control: Yamada,and Primbs. (2018).

- $\quad$ Multi period Model: Zhou,et al. (2018).

- $\quad$ Operational Research: Zhao, Chakrabarti,and Muthuraman. (2019).

- $\quad$ Operational Risk: Eckert, Gatzert and Heidinger. (2020).

- $\quad$ Portfolio Analysis: Zhu, Yu, and Mathew. (2020).

- $\quad$ Regression: Filip Klimenka \& James Lewis Wolter (2019).

- $\quad$ Replica Analysis: Shinzato. (2016).

- $\quad$ Robust Optimization: Adhikari, Putnam, and Panta. (2020).

- $\quad$ Sampling Resampling Monte Carlo: Shadabfar, and Cheng. (2020).

- $\quad$ Shrinkage: Sun, Ma, and Liu. (2018).

- $\quad$ Social Models: Torres, and Enciso (2017).

- $\quad$ Stochastic Models: Post, Karabatiand Arvanitis. (2018).

- $\quad$ Utilities: Lai. et al. (2019).

- $\quad$ Value-at-Risk: Zaichao Du, and Pei. (2020). 
E continuando na Tabela 30, as referências são:

- $\quad$ Adaptive models: Ruidi Song and Yue Chan (2020

- $\quad$ Bayesian models: Becker, Gurtler, Hibbelin . (2015).

- $\quad$ Black-Litterman: Xinxin, Jianjun. (2016).

- Bootstrap: Begoña. (2016).

- $\quad$ Cluster models: Mahdi, Masoud, Alireza A. (2020).

- $\quad$ Conditional value-at-risk: Christopher, Millery. (2017).

- $\quad$ Copula models: Kwong, Low. (2015).

- Data envelopment analysis: Edirisinghe, Zhang. (2010).

- $\quad$ Diversification: DeMiguel, Garlappi, Uppal. (2009).

- Diversos: Kolm, Tütüncü, Fabozzi. (2014).

- Downside models: Fortin, Jaroslava Hlouskova. (2015).

- Dynamic optimization: Bianchi, Guidoliny. (2013).

- $\quad$ Forecast: Allen, Lizieri, Satchell. (2019).

- $\quad$ Foreign studies: Owen. (2015).

- $\quad$ Fuzzy models: Kaczmarek, Dymova, Sevastjanov. (2020).

- $\quad$ Garch models: Aziz, Spyridon Vrontos, Hasim. (2019).

- $\quad$ Genetic Algorithm. (2012).

- Geometric Brownian: Abensur, Moreira, Faria. (2020).

- Interval selection: Li, Qin. (2014).

- $\quad$ Learning models: Ban, Karoui, Lim. (2016).

- $\quad$ Linear programming: Yu, Chiou, Mu. (2015).

- Mean models: Banihashemi, Azarpour, Navvabpour. (2016).

- Multicriteria models: Santamaría, Aguarón, Moreno-Jiménez. (2020).

- Multiobjective models: Hilario-Caballero. et al. (2020).

- $\quad$ Multi period models: Liu, Zhang, Zhang. (2013).

- $\quad$ Multivariate models: Bianchi, Tassinari. (2018).

- $\quad$ Nonparametric: Salah. et al. (2018).

- $\quad$ Performance measures: Caporin. (2014).

- $\quad$ Robust optimization: Tang, Ling. (2014).

- Sharpe ratio: Wang, Chen, Lianet al. (2020).

- $\quad$ Shrinkage: DeMiguel, Martin-Utrera, Nogales. (2013).

- $\quad$ Stochastic models: Post, Karabat1, Arvanitis. (2018).

- $\quad$ Tail dependence: Leal, Mendes. (2013).

- Value-at-risk: Du, Pei. (2020).

- Variance: Sui, Hu, Ma. (2020).

- Vast Portfólio: Fan, Zhang, Yu. (2012). 
Analisando as Tabela 29 e Tabela 30, e confirmando o que foi identificado principalmente na Figura 6, na Tabela 19 e na Figura 8 acima, as conclusões da análise bibliométrica em relação às duas suposições, sobre as evoluções, até a presente data, dos modelos da teoria de portfólios E-V eficientes de Markowitz, apresentadas neste trabalho são que:

- $\quad$ A simulação vem sendo utilizada nos modelos de otimização de portfólios como um meio para se obter melhores resultados e modelos, para se validar resultados, já que problemas práticos podem ser inacessíveis para modelagem exceto quando se recorre à simulação, como um meio para comparação entre modelos ou resultados, como análise de resultados dos modelos ou ainda para projeção dos resultados dos modelos em janelas de holdout, porém, com a apresentação dos resultados sendo sempre realizada pelo método de concentração de dados. Não foi identificado, em nenhum dos documentos revisados na pesquisa bibliométrica realizada, a simulação sendo utilizada nos modelos de otimização de portfólios como um fim, ou seja, para obter a distribuição de probabilidade empírica de todos os resultados ótimos inerentes à simulação.

As pesquisas bibliométricas realizadas não apresentaram em seus resultados uma métrica do risco na forma de uma métrica do risco com espaço objetivo aumentado, de forma a considerar um maior número de parâmetros a serem otimizados, possibilitando, assim, melhores resultados. Não foi identificado em nenhum dos documentos revisados na pesquisa bibliométricas realizada métricas do risco com o espaço objetivo aumentado.

\section{Conclusão}

Com o estudo se pode concluir que as ferramentas com o objetivo de auxiliar na busca por temas específicos se tornaram de extrema necessidade para mensurar e quantificar, de forma temporal, estudos relacionados aos assuntos de interesse dos pesquisadores. Com a análise bibliométrica, é possível sumarizar os dados relacionados as palavras chaves de interesse e conseguir observar, o número de publicações com o tema de interesse no período escolhido, assim como os autores que mais produzem, as quantidades de citações, a colaboração entre países, o autor mais citado, o jornal que mais publicou artigos com o tema, dentre outros. Como demonstrado também é possível realizar a validação ou a rejeição a respeito de suposições da existência de soluções específicas para determinada área de pesquisa. Verificamos assim que a bibliometria é de grande eficiência e utilidade quando se busca por levantamentos científicos em massa. A bibliometria é de fato uma ferramenta essencial e a mesma vem sendo utilizada de forma cada vez mais frequente, juntamente com os programas e softwares que possibilitam a sua realização. Somente pelo uso da bibliometria é possível chegar as conclusão de que, até a presente data: primeiro, a simulação vem sendo utilizada nos modelos de otimização de portfólios como um meio para se obter melhores resultados e modelos, para se validar resultados, já que problemas práticos podem ser inacessíveis para modelagem exceto quando se recorre à simulação, como um meio para comparação entre modelos ou resultados, como análise de resultados dos modelos ou ainda para projeção dos resultados dos modelos em janelas de holdout, porém, com a apresentação dos resultados sendo sempre realizada pelo método de concentração de dados. Não foi identificado, em nenhum dos documentos revisados na pesquisa bibliométrica realizada, a simulação sendo utilizada nos modelos de otimização de portfólios como um fim, ou seja, para obter a distribuição de probabilidade empírica de todos os resultados ótimos inerentes à simulação e segundo, as pesquisas bibliométricas realizadas não apresentam em seus resultados uma métrica do risco na forma de uma métrica do risco com espaço objetivo aumentado, de forma a considerar um maior número de parâmetros a serem otimizados, possibilitando, assim, melhores resultados. Não foi identificado em nenhum dos documentos revisados nas pesquisas bibliométricas realizadas métricas do risco com o espaço objetivo aumentado. Assim é demonstrado o grande potencial da bibliometria e de forma que se pode fortemente sugerir que artigos futuros tenham sempre sua pesquisa bibliográfica acompanhada de um estudo bibliométrico a respeito do assunto abordado pela pesquisa do artigo. 


\section{Agradecimentos}

Agradecemos a DBD - Divisão de Bibliotecas e Documentação da PUC-Rio - pelo auxílio na utilização dos repositórios de dados utilizados e a Iasmyn Lugon pela realização das figuras.

\section{Referências}

Abensur, E. O., Moreira, D. F., \& Faria A. C. R. (2020). GeometricC Brownian Motion: An Alternative to High-Frequency Trading for Small Investors. Independent Journal of Management \& Production (IJM\&P), 10.14807/ijmp.v11i3.1114. http://www.ijmp.jor.br.

Adhikari, R., Putnam, K. J., \& Panta, H. (2020). Robust Optimization-Based Commodity Portfolio Performance. Internacional Jounal of. Financial Studies, 8 , p. $54,2020$.

Allen, D., Lizieri, C., \& Satchell, S. (2019). In Defense of Portfolio Optimisation What If We Can Forecast? https://ssrn.com/abstract=3373594.

Andrade, F. (2012). Análise Bibliométrica da produção científica de pesquisadores e referências de um período da engenharia de produção. Universidade Federal do Rio Grande do Sul, 2012.

André, C. (2012) Análise Bibliométrica do Periódico Científico Trans informação. Universidade Federal de Santa Catarina, 2012.

Araújo, C. (2006). Bibliometria: evolução histórica e questões atuais. Em Questão, 12(1), 11-32. 2006.

Aziz, N. S. A., Spyridon V., S., \& Hasim, H. M. (2019). Evaluation of multivariate GARCH models in an optimal asset allocation framework. North American Journal of Economics and Finance, 47 (2019) 568-596.

Bahia, E., Santos, R., \& Blattmann, U. (2011). Estudo Bibliométrico sobre preservação digital: Library and information science abstracts - LISA. Enc. Bibli: R. Eletr. Bibliotecon. Ci. Inf..

Ban, G., Karoui, N. E., \& Lim, A. E. B. (2016). Machine Learning and Portfolio Optimization. Published online in Management Science Articles in Advance, 21 Nov 2016. http://dx.doi.org/10.1287/mnsc.2016.2644.

Banihashemi, S., Azarpour, A. M., \& Navvabpour, H. (2016). Portfolio Optimization by Mean-Value at Risk Framework. Applied Mathematics \& Information Sciences, 10(5), 1935-1948 (2016).

Becker, F., Gurtler, M., \& Hibbelin M. (2015). Markowitz versus Michaud Portfolio Optimization Strategies Reconsidered. (2015), European Journal of. Finance, 21, 269-291.

Begoña F. (2016). Bootstrap estimation of the efficient frontier. Computer Management Science, (2016) 13:541-570. 10.1007/s10287-016-0257-2.

Begusiae, S. \& Kostanjèar, Z. (2020). Cluster-Specific Latent Factor Estimation in High-Dimensional Financial Time Series. in IEEE Access, 8, 164365164379, 2020, 10.1109/ACCESS.2020.3021898.

Bianchi, D., \& Guidoliny, M. (2013). Can Long-Run Dynamic Optimal Strategies Outperform Fixed-Mix Portfolios? Evidence from Multiple Data Sets. http://ssrn.com/abstract=2353761.

Bianchi, M. L., \& Tassinari, G. L. (2018). Forward-looking portfolio selection with multivariate non-Gaussian models and the Esscher transform. arXiv:1805.05584v2.

Caporin, M. (2014). A Survey on the Four Families of Performance Measures. Journal of Economic Surveys, (2014) 28(5), 917-942.

Café, L., \& Brascher, M. (2008). Organização da Informação e Bibliometria. Enc. Bibli: R. Eletr. Bibliotecon. Ci. Inf..

Chakkalakal, L., Hommel, U., \& Li, W. (2018). Transport infrastructure equities in mixed-asset portfolios: estimating risk with a Garch-Copula CVaR model, Journal of Property Research, 35: 2, 117-138, 10.1080/09599916.2018.1461126.

Christopher, W., \& Millery, I. Y. (2017). Optimal Control of Conditional Value-at-Risk in Continuous Time. arXiv:1512.05015v3.

Chueke, G., \& Amatucci, M. (2015). O que é bibliometria? Uma introdução ao Fórum. 11(2), 1-5. 2015.

Cong, F., \& Oosterlee, C. W. (2016). Multi-period mean-variance portfolio optimization based on Monte-Carlo simulation. Journal of Economic Dynamics \& Control, 64(2016)23-38.

DeMiguel, V., Garlappi, L., \& Uppal, R., (2009). Optimal Versus Naive Diversification: How Inefficient is the 1/N Portfolio Strategy? The Review of Financial Studies 22(5)2009. 10.1093/rfs/hhm075.

DeMiguel, V., Martin-Utrera, A., \& Nogales, F. J. (2013). Size matters: Optimal calibration of shrinkage estimators for portfolio Selection. Journal of Banking \& Finance, 37 (2013) 3018-3034.

Domingues, M. A. (2018). Mapeamento da Ciência com o Pacote R Bibliometrix: Uma aplicação no estudo de Empreendedorismo Acadêmico. Proceeding of ISTI/SIMTEC (2018), 10.7198/S2318-3403201800010033, 9(1), 287-294.

Du, Z., \& Pei, P. (2020). Backtesting Portfolio Value-at-Risk with Estimated Portfólio Weights. Journal of. Time Series. Analysis, 41: 605-619 (2020). Published online 06April 2020 inWileyOnline Library (wileyonlinelibrary.com) 10.1111/jtsa.12524. 
Eckert, C., Gatzert, N., \& Heidinger, D. (2020). Empirically assessing and modeling spillover effects from operational risk events in the insurance industry. Insurance: Mathematics and Economics, 93, 72-83, 2020.

Edirisinghe, N. C. P., \& Zhang, X. (2010). Input/output selection in DEA under expert information with application to financial markets. European Journal of Operational Research 207 (2010) 1669-1678.

Ekblom, J., \& Blomvall J. (2020). Importance sampling in stochastic optimization: An application to intertemporal portfolio choice. European Journal of Operational Research, 285, 106-119, 2020.

Fan, J., Zhang, J., \& Yu, K. (2012). Vast Portfolio Selection with Gross-Exposure Constraints. Journal of the American Statistical Association, 107: 498, 592606.

Fortin, J. H. J. (2015). Downside loss aversion:Winner or loser? Math Meth Oper Res, (2015) 81:181-233. 10.1007/s00186-015-0493-1.

Hilario-Caballero, A. et al. (2020). Tri-Criterion Model for Constructing Low-Carbon Mutual Fund Portfolios: A Preference-Based Multi-Objective Genetic Algorithm Approach. International Journal of Environmental Research and. Public Health, 17, 6324, 10.3390/ijerph17176324.

Huang, S., \& Lin, T. (2018). A Linearization of the Portfolio Optimization Problem with General Risk Measures Under Multivariate Conditional Heteroskedastic Models. Asia-Pacific Journal of Financial Studies, 47, 449-469. 10.1111/ajfs.12218.

Jiang, G., Hong, L. J., \& Nelson, B. L. (2020). Online Risk Monitoring Using Offline Simulation. INFORMS Journal on Computing, 32(2), 356-375. https://doi.org/10.1287/ijoc.2019.0892.

Kaczmarek, K., Dymova, L., \& Sevastjanov, P. (2020). A Simple View on the Interval and Fuzzy Portfolio Selection Problems. Entropy, 22, 932, $10.3390 / \mathrm{e} 22090932$.

Klimenka, F., \& Wolter, J. L. (2019). Multiple Regression Model Averaging and the Focused Information Criterion with an Application to Portfolio Choice. Journal of Business \& Economic Statistics, 37: 3, 506-516, 10.1080/07350015.2017.1383262.

Kolm, P. N., Tütüncü, R., \& Fabozzi, F. J. (2014). 60 Years of portfolio optimization: Practical challenges and current trends. European Journal of Operational Research 234 (2014) 356-371.

Kwong, R., \& Low, Y. (2015). Vine copulas: Modeling systemic risk and enhancing higher-moment portfolio optimization. http://ssrn.com/abstract=2259076.

Lai, S. et al, (2019). Gas Generation Portfolio Management Strategy Based on Financial Derivatives: Options. 2019 9th International Conference on Power and Energy Systems (ICPES), 1-6, 10.1109/ICPES47639.2019.9105461.

Leal, R. P. C., \& Mendes, B. V. M. (2013). Assessing the effect of tail dependence in portfolio allocations. Applied Financial Economics, 2013 23(15), 12491256, http://dx.doi.org/10.1080/09603107.2013.804160.

Li, X., \& Qin, Z. (2014). Interval portfolio selection models within the framework of uncertainty theory. Economic Modelling 41 (2014) 338-344.

Lin, P. (2012). Portfolio Optimization and Risk Measurement Based on Non-Dominated Sorting Genetic Algorithm. Journal of Industrial and Management Optimization, 8(3), 10.3934/jimo.2012.8.549.

Liu, Y., Zhang, W., \& Zhang, P. (2013). A multi-period portfolio selection optimization model by using interval analysis. Economic Modelling, 33 (2013) 113119.

Mahdi, M., Masoud, M., \& Alireza A., K., (2020). Development of an efficient cluster-based portfolio optimization model under realistic market conditions. Empirical Economics, (2020), 59:2423-2442. https://doi.org/10.1007/s00181-019-01802-5.

Mello, I., Barbosa, K., Dantas, J., \& Botelho, D. (2015). 25 Anos de Publicação em Auditoria: Análise Bibliométrica com Ênfase na Lei de Lotka, Lei de Zipf e Ponto de Transição (T) de Goffman. Congresso de Contabilidade, Universidade Federal de Santa Catarina. 2015.

Mugnaini, R. (2004). Indicadores bibliométricos da produção científica brasileira: uma análise a partir da base Pascal, Ci. Inf., Brasília, 33(2), $123-131,2004$.

Münnix, M. C., Schäfer, R., \& Grothe, O. (2014). Estimating correlation and covariance matrices by weighting of market similarity. Quantitative Finance, 14: 5, pp. 931-939, 10.1080/14697688.2011.605075.

Naccarato, A., \& Pierini, A. (2014). Element-by-element estimation of a volatility matrix. An Italian portfolio simulation. Investment Management and Financial Innovations, 11(3), 2014.

Otlet, P. (1934). Traité de documentatión: Le Livre Sur Le Livre - Théorie et Pratique. Éditeurs-Imprimeurs D. Van Keerberghen \& Fils, Editones Mundaneum, Palais Mondial. https://lib.ugent.be/fulltxt/handle/1854/5612/Traite_de_documentation_ocr.pdf.

Owen W. S. (2015). Foreign Currency Exposure within Country Exchange Traded Funds. Frontiers in Finance, 1, 2015.

Penteado, R. (2002). Aplicação da Bibliometria na Construção de Indicadores sobre a Produção Científica da Embrapa. Workshop Brasileiro de Inteligência Competitiva e Gestão do Conhecimento. 2002.

Pimenta, A., Portela, A., Oliveira, C., \& Ribeiro, R. (2017). A Bibliometria nas pesquisas acadêmicas. Revista de ensino, pesquisa e extensão, 4(7), 2017.

Pizzani, L., Silva, R., \& Hossne, W. (2010). Análise bibliométrica dos 40 anos da produção científica em Bioética no Brasil e no mundo. Revista - Centro Universitário São Camilo. 2010. 
Platanakis, E., Sutcliffe, C., \& Ye, X. (2020). Horses for Courses: Mean-Variance for Asset Allocation and 1/N for Stock Selection. https://ssrn.com/abstract=3372334.

Post, T., Karabati, S., \& Arvanitis, S. (2018). Portfolio optimization based on stochastic dominance and empirical likelihood. Journal of Econometrics, 206 (2018) 167-186.

Pritchard, A. (1969). Statistical bibliography or bibliometrics? Journal of Documentation, 24(4), 348-349. SCOPUS. https://wwwscopus.ez20.periodicos.capes.gov.br/search/form.uri?display=basic.

Resta, M. (2012). Portfolio optimization with dimension reduction techniques: A comprehensive simulation study. Neurocomputing: Learning, Architectures and Modeling, 9, 93-118, 2012.

Ruidi S., \& Yue C. (2020). A New Adaptive Entropy Portfolio Selection Model. Entropy. 22, 951, 10.3390/e22090951.

Salah, H. B. et al. (2018). Mean and median-based nonparametric estimation of returns in mean-downside risk portfolio frontier. Ann Oper Res., (2018) 262:653681. https://doi.org/10.1007/s10479-016-2235-z.

Santamaría, R., Aguarón, J., \& Moreno-Jiménez, J. M. (2020). A multicriteria approach based on Analytic Hierarchy Process and compromise programming in portfolio selection. J Multi-Crit Decis Anal. 2020, 27, 141-146. 10.1002/mcda.1699.

Scarpel, L. (2016). Pesquisa Científica. Instituto Tecnológico de Aeronáutica Engenharia Mecânica-Aeronáutica (IEM), Departamento de gestão e suporte à decisão centro de gestão em engenharia. 2016.

Shadabfar, M., \& Cheng, L. (2020). Probabilistic approach for optimal portfolio selection using a hybrid Monte Carlo simulation and Markowitz model. Alexandria Engineering Journal, 59, 3381-3393, 2020.

Shen, W. et al. (2019). The Kelly Growth Optimal Portfolio with Ensemble Learning. The Thirty-Third AAAI Conference on Artificial Intelligence (AAAI-19).

Shinzato, T. (2016). Replica Analysis for the Duality of the Portfolio Optimization Problem. arXiv:1609.05475v1.

Silva, F., Santos, B. M. E., \& Vils, L. (2016). Estudo Bibliométrico: Orientações sobre sua Aplicação. Revista Brasileira de Marketing, 2016.

Soares, P., Carneiro, T., Calmon, Castro, J., \&Otávio L. (2016). Análise bibliométrica da produção científica brasileira sobre Tecnologia de Construção e Edificações na base de dados Web of Science. Ambiente Construído, Porto Alegre, 16(1), 175-185. 2016.

Sui, Y., Hu, J., \& Ma, F. (2020). A Mean-Variance Portfolio Selection Model with Interval-Valued Possibility Measures. Mathematical Problems in Engineering, 12. https://doi.org/10.1155/2020/4135740.

Sun, R., Ma, T., \& Liu, S. (2018). A Stein-type shrinkage estimator of the covariance matrix for portfolio selections. Metrika (2018), 81, 931-952. https://doi.org/10.1007/s00184-018-0663-2.

Tang, L., \& Ling, A. (2014). Closed-Form Solution for Robust Portfolio Selection with Worst-Case CVaR Risk Measure. Mathematical Problems in Engineering, Volume 2014, Article ID 494575, 9 pages. http://dx.doi.org/10.1155/2014/494575.

Torres, O. T., \& Enciso, I. M. T (2017). Is socially responsible investment useful in Mexico? A multi-factor and ex-ante review. Contaduría y Administración, 62 (2017) pp. 222-238

Vasyl, G. V., et al. (2020). Statistical inferences for realized portfolio weights. Econometrics and Statistics, 14, 49-62, 2020.

Vanti, N. A. P. (2002) Da bibliometria à webometria: uma exploração conceitual dos mecanismos utilizados para medir o registro da informação e a difusão do conhecimento. Ciência da Informação, Brasília, 31(2), 152-162.

Wallin, M. W. The bibliometric structure of spin-off literature. Innovation: Management, Policy, \& Practice, 14(2), 162(16), 2012.

Wang, C. D., et al. (2020). Asset selection based on high frequency Sharpe ratio. Journal of Econometrics (2020), https://doi.org/10.1016/j.jeconom.2020.05.007.

Xiao, H., Ren, T., \& Ren, T. (2020). Estimation of fuzzy portfolio efficiency via an improved DEA approach. INFOR: Information Systems and Operational Research, 58: 3, pp. 478-510, 10.1080/03155986.2020.1734904.

Xinxin, J., \& Jianjun, G. (2016). Extensions of Black-Litterman portfolio optimization model with downside risk measure. IEEE, 978-1-4673-9714$8 / 16 / \$ 31.00 @ 2016$.

Yamada, Y., \& Primbs, J.A. (2018). Model Predictive Control for Optimal Pairs Trading Portfolio with Gross Exposure and Transaction Cost Constraints. Asia-Pacific Finan Markets, 25. 1-21, 2018. https://doi.org/10.1007/s10690-017-9236-z.

Yan, D., Hu, Y. \& Lai, K. A. (2018). A Nonlinear Interval Portfolio Selection Model and Its Application in Banks. J Syst Sci Complex 31, 696-733 (2018). https://doi.org/10.1007/s11424-017-6070-3.

Yu, J., Chiou, W. P., \& Mu, D. (2015). A linearized value-at-risk model with transaction costs and short selling. European Journal of Operational Research 247 (2015) 872-878.

Yua, X. et al. (2020). Hedging the exchange rate risk for international portfolios. Mathematics and Computers in Simulation, 173, 85-104, 2020.

Zhao, L., Chakrabarti, D., \& Muthuraman, K. (2019). Portfolio Construction by Mitigating Error Amplification: The Bounded-Noise Portfolio. Operations Research, 67(4): 965-983. https://doi.org/10.1287/opre.2019.1858. 
Research, Society and Development, v. 10, n. 4, e57310413802, 2021

(CC BY 4.0) | ISSN 2525-3409 | DOI: http://dx.doi.org/10.33448/rsd-v10i4.13802

Zhou, Z. et al. (2018). Time-Consistent Strategies for Multi-Period Portfolio Optimization with/without the Risk-Free Asset. Mathematical Problems in Engineering, 20 https://doi.org/10.1155/2018/7563093.

Zhu, Y., Yu, P. L. H., \& Mathew, T. (2020). Improved estimation of optimal portfolio with an application to the US stock market. Journal of Statistical Theory and Practice, 14(1). https://doi.org/10.1007/s42519-019-0067-2. 